\title{
A New Mechanism for Generating a Single Transverse Spin Asymmetry
}

\author{
Yuri V. Kovchegov国 Matthew D. Sievert回 \\ Department of Physics, The Ohio State University, Columbus, OH 43210, USA
}

\begin{abstract}
We propose a new mechanism for generating a single transverse spin asymmetry (STSA) in polarized proton-proton and proton-nucleus collisions in the high-energy scattering approximation. In this framework the STSA originates from the $q \rightarrow q G$ splitting in the projectile (proton) light-cone wave function followed by a perturbative $(C$-odd) odderon interaction, together with a $C$-even interaction, between the projectile and the target. We show that some aspects of the obtained expression for the STSA of the produced quarks are in qualitative agreement with experiment: STSA decreases with decreasing projectile $x_{F}$ and is a non-monotonic function of the transverse momentum $k_{T}$. In our framework the STSA peaks at $k_{T}$ near the saturation scale $Q_{s}$. Our mechanism predicts that the quark STSA in proton-nucleus collisions should be much smaller than in proton-proton collisions. We also observe that in our formalism the STSA for prompt photons is zero.
\end{abstract}

PACS numbers: 24.85.+p, 12.38.Bx, 13.88.+e, 24.70.+s

\section{INTRODUCTION}

In recent years a significant theoretical effort has been directed toward understanding the origin and the interesting properties of transverse spin asymmetries observed in high-energy scattering experiments. The single transverse spin asymmetry (STSA) in polarized scattering $P^{\uparrow}(p)+A \rightarrow h(k)+X$ is an observable describing the correlation between the transverse spin vector $\boldsymbol{S}$ of the projectile $P$ and the transverse momentum $\boldsymbol{k}$ of the produced hadron $h$. This correlation can be expressed either in terms of the asymmetry in the scattering of the spin-up and spin-down transverse spin states, or in terms of the "left-right" $(\boldsymbol{k} \leftrightarrow-\boldsymbol{k})$ asymmetric momentum distribution in spin-up scattering:

$$
A_{N}(\boldsymbol{k}) \equiv \frac{\frac{d \sigma^{\uparrow}}{d^{2} k d y}-\frac{d \sigma^{\downarrow}}{d^{2} k d y}}{\frac{d \sigma^{\uparrow}}{d^{2} k d y}+\frac{d \sigma^{\downarrow}}{d^{2} k d y}}=\frac{\frac{d \sigma^{\uparrow}}{d^{2} k d y}(\boldsymbol{k})-\frac{d \sigma^{\uparrow}}{d^{2} k d y}(-\boldsymbol{k})}{\frac{d \sigma^{\uparrow}}{d^{2} k d y}(\boldsymbol{k})+\frac{d \sigma^{\uparrow}}{d^{2} k d y}(-\boldsymbol{k})} \equiv \frac{d(\Delta \sigma)}{2 d \sigma_{u n p}} .
$$

Interpreted as a left-right asymmetry in the produced hadron distribution, the sign of $A_{N}$ is fixed to reflect a preferential scattering of particles to the beam-left when $A_{N}$ is positive and a preferential scattering to the beam-right when $A_{N}$ is negative. More concretely, if the polarized projectile moves along the $+z$ axis and the transverse spin is oriented along the $+x$ axis, then positive $A_{N}$ corresponds to more outgoing particles produced along the $-y$ axis than along the $+y$ axis. The geometry of polarized scattering is illustrated in Fig. 1 The STSA can also be thought of as a spin-momentum coupling term proportional to $(\vec{S} \times \vec{p}) \cdot \vec{k}$ in the particle production cross section, where $\vec{p}$ is the momentum of the incoming projectile. For a review of the current status of STSA physics see [1].

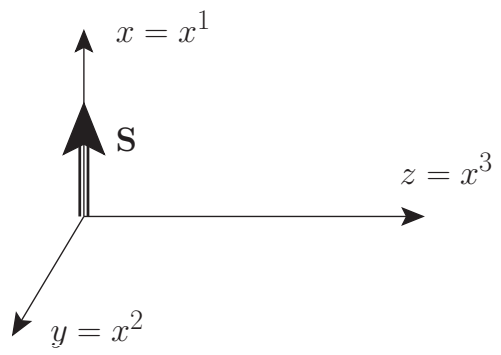

FIG. 1. A sketch of the geometry of polarized scattering: the incoming transversely-polarized projectile is moving along the $+z$-axis, while its spin is pointing along the $+x$-axis. The positive $y$-axis points to the right of the beam.

In the 1990's, the E581 and E704 collaborations at Fermilab reported data on hadron production in transversely polarized proton-proton scattering that showed large unanticipated transverse spin asymmetries of up to $30-40 \%$ [27]. Qualitatively, the asymmetries were consistent with zero for mid rapidities, but increased rapidly in the forward

\footnotetext{
a kovchegov.1@asc.ohio-state.edu

b sievert.7@osu.edu
} 

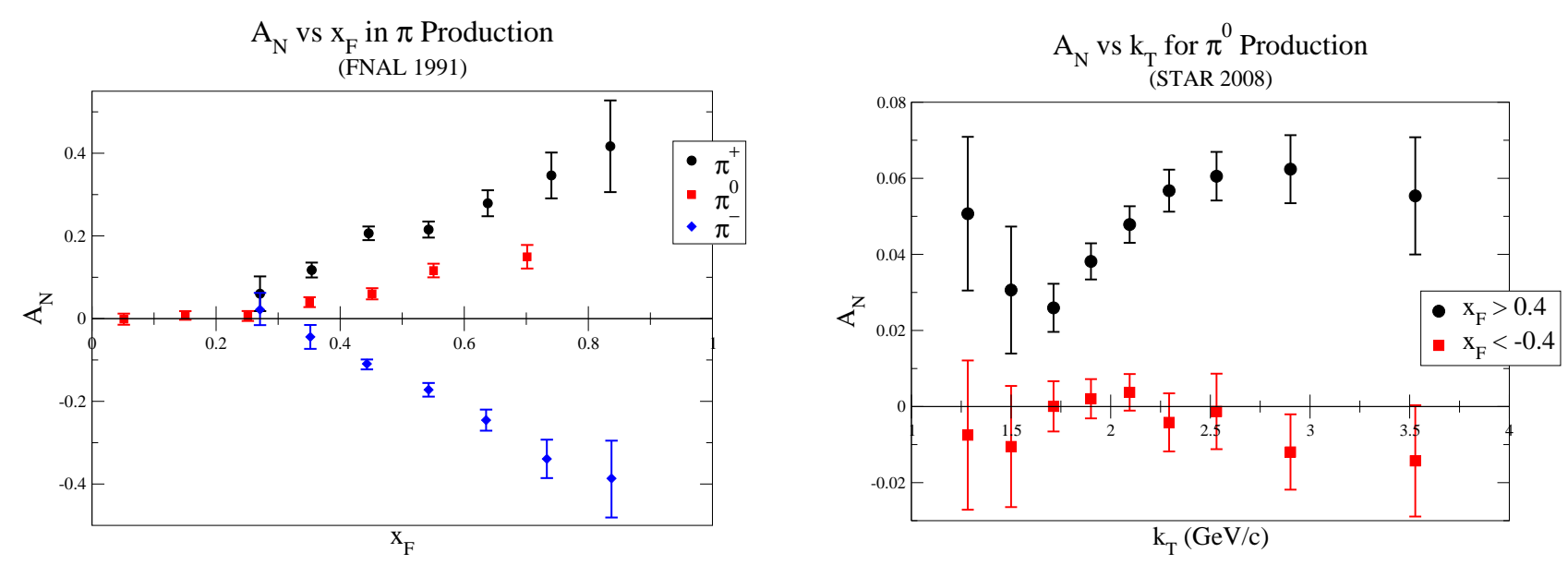

FIG. 2. Experimental data on the pion single transverse spin asymmetry $A_{N}$ as a function of Feynman- $x$ reported by E581 and E704 collaborations (graphically reconstructed from [3], shown in the left panel) for $0.7 \leq k_{T} \leq 2.0 \mathrm{GeV} / \mathrm{c}$, and as a function of the pion transverse momentum $k_{T}$ collected by the STAR collaboration [12] (right panel).

scattering direction, as illustrated by the data shown in the left panel of Fig. 21 More recently, the PHENIX, STAR, and BRAHMS collaborations at the Relativistic Heavy Ion Collider (RHIC) have studied transverse spin asymmetries over a wide kinematic range at $\sqrt{s}=200 \mathrm{GeV}[8-11]$. The data they have presented [8 10] confirmed and extended the Fermilab results, and also indicated a non-monotonic dependence of STSA on the transverse momentum $k_{T}$ of the produced hadron [12, 13], shown in the right panel of Fig. [2.

At the time these spin asymmetries were first observed, there was no theoretical framework to understand them; on the contrary, prevailing wisdom expected spin-dependent effects to become negligible at high energies [14]. Much theoretical progress in classifying and modeling sources of STSA has been made since then, predominantly within the framework of collinear factorization.

There are three stages in the scattering process where an asymmetry could be generated, illustrated in Fig. 3 , Sivers 15, 16 proposed the existence of a correlation between the spin of the polarized hadron and the transverse momentum distribution of its partons. This Sivers effect would act within the parton distribution function (PDF) (or, equivalently, the initial state wave function) of the polarized projectile to generate an asymmetry. Being a part of the PDF, this is an intrinsically non-perturbative process. The asymmetry could also be generated in the partonic scattering processes themselves; since the leading-order scattering process does not generate an asymmetry, any contribution would come from "higher-twist" terms in the interaction, as suggested in 17 26]. Such an asymmetry generated by the interaction may be perturbative, depending on the kinematics. Finally, Collins [27] proposed a similar correlation between spin and transverse momentum that could occur during hadronization, as a produced parton undergoes fragmentation into final-state hadrons. The Collins effect would couple the spin of a produced parton to the momentum dependence of

$\underline{\text { Sivers Effect }}$

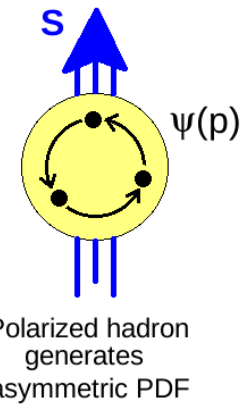

Interaction Effects

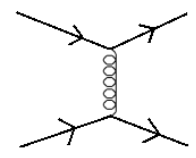

Parton-level asymmetric scattering
Collins Effect

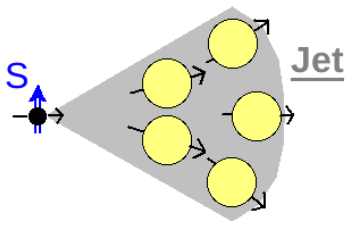

Polarized parton undergoes asymmetric fragmentation

FIG. 3. Sketch of the three potential sources of asymmetry: in the parton distribution function via the Sivers effect, via partonic interaction, or in the fragmentation function via the Collins effect. 
its fragmentation function, resulting in a left-right asymmetry within the final-state jet. Again, the Collins effect is a low-energy, intrinsically non-perturbative process.

Recent studies from STAR [28] were able to resolve the angular distribution within the jets and thus probe the Collins function directly. Their data suggests that the Collins effect's contribution to the asymmetry is small or consistent with zero, as illustrated in Fig. 4.

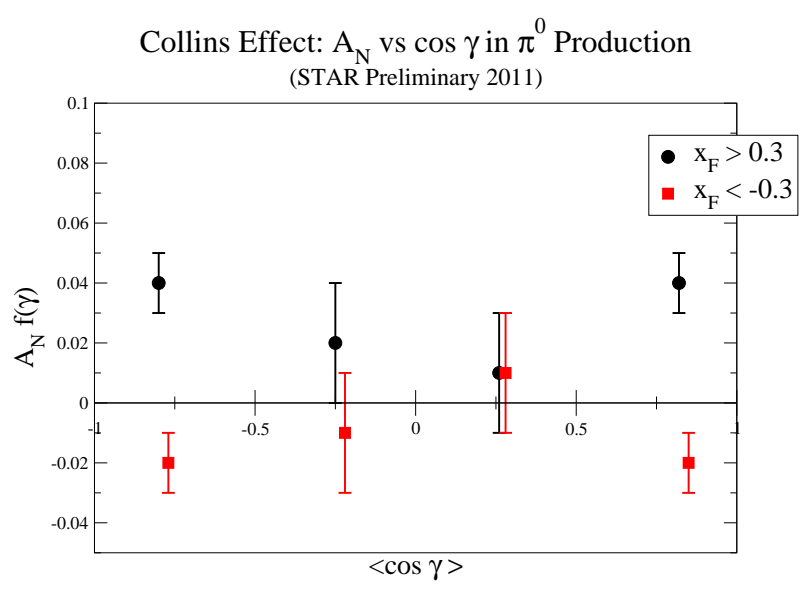

FIG. 4. Recent preliminary data from the STAR collaboration (graphically reconstructed from [28]) for the $\pi^{0}$ asymmetry within final-state jets as a function of the angle $\gamma$ between the outgoing pion and the jet thrust axis. The Collins contribution is proportional to the slope of the data, and is consistent with zero.

We are interested in understanding which perturbative mechanisms within the interaction can give rise to transverse spin asymmetries, and we would like to study the interaction with proton-nucleus $\left(p^{\uparrow}+A\right)$ collisions in mind. When the target is a heavy nucleus at high energies, or when the energy of the proton-proton collision is high enough, the projectile scatters off of the target's small- $x$ parton distribution where parton densities are high. The effective interactions in this small- $x$ regime are well described by the parton saturation/Color Glass Condensate (CGC) formalism [29], 30], 31], 32 34], 35 37], 38, 39], [40 48], [49 53], [54 56] which resums the multiple rescatterings in these dense color fields and incorporates the small- $x$ evolution of those fields. This saturation framework is expressed naturally in coordinate space and introduces a characteristic saturation scale $Q_{s}$ describing the color-charge density fluctuations within the target.

With the CGC interactions that mediate $p^{\uparrow}+A$ scattering in mind, it is natural to search for the asymmetry using the language of the small- $x$ interactions, light-cone perturbation theory (LCPT) [57, 58], instead of the usual collinear factorization. Using LCPT, we calculate spin-dependent processes that contribute to the projectile's light-cone wave function, then convolute this wave function (squared) with the known scattering amplitudes from CGC that are responsible for the interaction of partons with the target. Surprisingly enough this procedure yields a non-zero STSA for quark and gluon production in $p^{\uparrow}+A$ scattering. Our approach yields a different but complementary picture to the usual collinear factorization mechanisms for generating STSA and may provide new insight into the underlying physics for generating transverse spin asymmetry.

In the past the interplay of spin and small- $x$ evolution was investigated in [59] using the standard Feynman diagram approach. In more recent years the role of spin has also been investigated within the McLerran-Venugopalan (MV) model [35 37] in [60] and within Mueller's dipole model [32 34] in 61]. The transverse spin asymmetry in the CGC formalism was studied in 62 65], with the STSA being a completely non-perturbative effect on top of the perturbative CGC dynamics. Other non-perturbative approaches include using QCD instantons to generate STSA as discussed in [66].

In this paper, we present an analysis of STSA generated by the interaction of the spin-dependent LCPT wave function of the projectile with the target gluon field in the saturation/CGC framework. The paper is organized as follows. In Sec. II we derive the general expressions for the quark, gluon, and photon single transverse spin asymmetries in our formalism. These general results are given in Eqs. (32), (40) and (44). We show that to generate the asymmetry the projectile needs to interact with the target via a $C$-odd scattering amplitude, commonly known as the QCD odderon [67-74]. In Sec. III we simplify the interaction of the light-cone wave function with the target using the Glauber-Mueller approximation [75], and we present a closed-form estimate of the asymmetry for produced quarks. We find that a $C$-odd exchange alone is insufficient to generate the asymmetry, and needs to be accompanied by a $C$-even exchange for the asymmetry to be non-zero. We also see that the generated STSA $\left(A_{N}\right)$ is a non-monotonic function of transverse momentum $k_{T}$ and an increasing function of $x_{F}$, in qualitative agreement with the data shown 
in Fig. 2. Our rough estimates of the asymmetry are plotted in Figs. 13, 14 and 15, which, when compared to the data in Fig. 2, suggest that it may even be possible to achieve quantitative agreement with the data using our approach in a detailed phenomenological study. We conclude by summarizing our main results in Sec. IV]

\section{GENERAL RESULT: COUPLING SPIN TO INTERACTION C-PARITY}

In this paper we consider two processes contributing to the STSA of hadron production in the scattering of transversely polarized protons on an unpolarized target (proton or nucleus), $p^{\uparrow}+A \rightarrow h+X$ : quark production and gluon production. We will also consider STSA in prompt photon production, $p^{\uparrow}+A \rightarrow \gamma+X$. The proton's transverse spin is transmitted to a quark in its wave function (giving rise to the transversity distribution); for simplicity we consider the scattering of this transversely polarized quark on the target separately from other (spectator) quarks in the proton. This process is illustrated in Fig. 5. where the high energy interaction between the projectile quark and the target nucleus is schematically denoted by gluon exchanges.

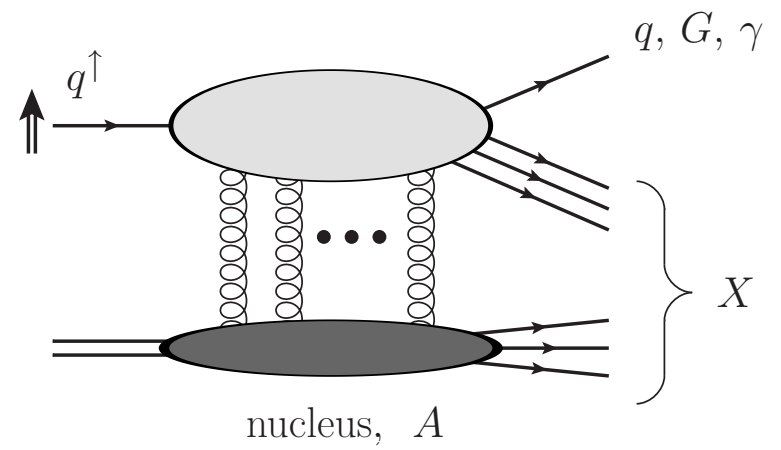

FIG. 5. Transversely polarized quark scattering in the field of the nucleus producing either a quark $(q)$, gluon $(G)$, or a prompt photon $(\gamma)$ along with extra hadrons denoted by $X: q^{\uparrow}+A \rightarrow(q, G, \gamma)+X$.

To introduce the methodology, we will concentrate on the quark production process, $q^{\uparrow}+A \rightarrow q+X$. We are interested in isolating the spin-dependent contribution of the quark-target scattering. Certainly keeping only the eikonal interaction would be insufficient to reach this goal, since the eikonal scattering is independent of the quark polarization. A non-eikonal correction has to be included: in the multiple-rescattering Glauber-Mueller [75] approximation, the non-eikonal rescattering corrections are suppressed by powers of energy and are very small. A much larger spin-dependent contribution comes from the non-eikonal splitting of the projectile quark into a quark and a gluon, $q \rightarrow q G$, which is suppressed only by a power of the strong coupling $\alpha_{s}$. In the LCPT language the $q \rightarrow q G$ splitting may take place either before or after the interaction with the target, as shown in Fig. 6. (Splitting during the interaction with the target is suppressed by powers of energy [6].) The lowest-order diagrams shown in Fig. 6 that contribute to STSA in $q^{\uparrow}+A \rightarrow q+X$ contain the emission of a single gluon from the polarized quark, where both the gluon and quark can scatter in the field of the target. (Multi-gluon non-eikonal emissions are also possible, but they are higher-order in $\alpha_{s}$ and, hence, outside of the leading-order precision of this work.) The spin dependence of the process illustrated in Fig. 6] originates within the light-cone wave function of the quark-gluon system, which couples to the interaction in a way that generates the asymmetry. In this Section, we will first outline the calculation of the $q \rightarrow q G$ light-cone wave function using LCPT. Then we will combine the resulting splitting wave function squared with the quark and gluon interactions in the field of the target and identify the contribution to the asymmetry. In the end we obtain general expressions for quark, gluon, and photon STSA's in our formalism. The incoming light quark has a particular flavor $f$; multiple quark flavors can be incorporated into our formalism by convoluting the obtained cross sections with quark distributions corresponding to different flavors (inserting the appropriate quark masses into our results below).

Throughout this paper, we will work in light-cone coordinates $p^{\mu}=\left(p^{+}, p^{-}, \boldsymbol{p}\right)$ with $p^{ \pm} \equiv p^{0} \pm p^{3}$ and the transverseplane vector $\boldsymbol{p} \equiv\left(p^{1}, p^{2}\right)$. Note that $p \cdot q=(1 / 2)\left(p^{+} q^{-}+p^{-} q^{+}\right)-\boldsymbol{p} \cdot \boldsymbol{q}$ and $p_{\mu} p^{\mu}=p^{+} p^{-}-p_{T}^{2}$ with $p_{T}=|\boldsymbol{p}|$. We assume that the incoming projectile quark is moving along the light-cone $x^{+}$-axis, while the target is moving along the $x^{-}$-axis. We will work in the light-cone gauge of the projectile, $A^{+}=0$. 

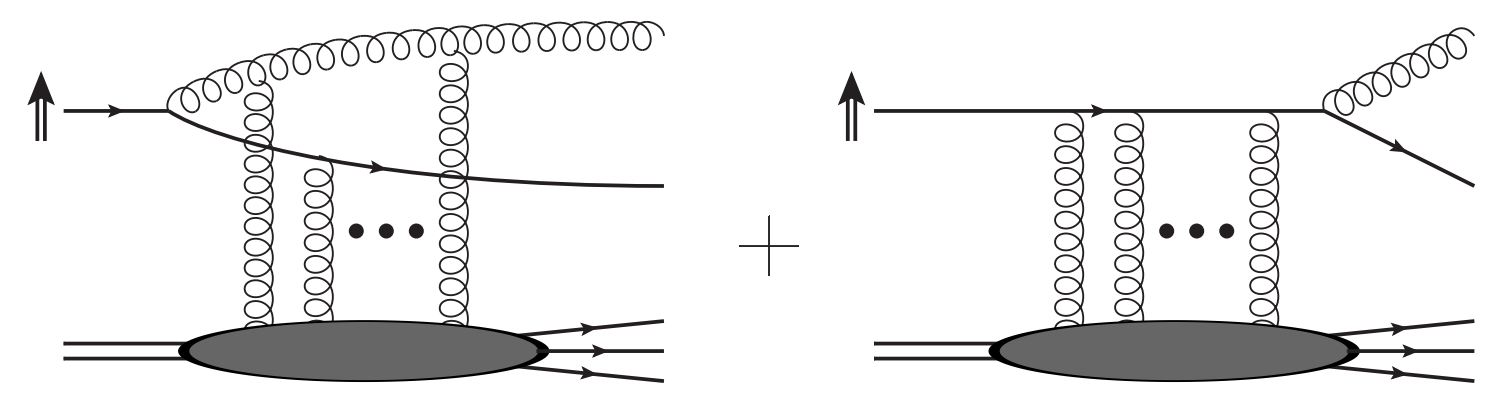

FIG. 6. Two contributions to the amplitude for the high energy quark-target scattering in LCPT.

\section{A. Light-Cone Wave Function and Transverse Polarization}

Consider the splitting shown in Fig. 7 of a transversely polarized quark with momentum $p$ and polarization $\chi= \pm 1$ decaying into a gluon (with momentum $p-k$, polarization $\lambda$, and color $a$ ) and a recoiling quark (with momentum $k$ and polarization $\chi^{\prime}$ ). The projectile quark is traveling along the light-cone $x^{+}$-direction and the recoiling quark carries a fraction

$$
\alpha \equiv \frac{k^{+}}{p^{+}}
$$

of the incoming quark's longitudinal momentum. We do not restrict ourselves to the case of an eikonal quark emitting a soft gluon $(1-\alpha \ll 1)$, but work in the general case when both the quark and the gluon can carry comparable longitudinal momenta.

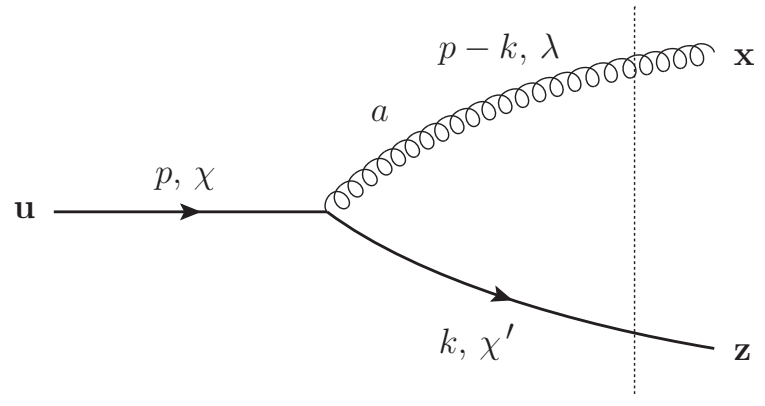

FIG. 7. The light-cone wave function for the $q \rightarrow q G$ splitting. Vertical dotted line denotes the intermediate state.

The calculation of the light-cone wave function corresponding to the diagram in Fig. 7 is different from other similar calculations in the literature (see e.g. 77, 78]) only in that now the incoming quark is polarized transversely. To account for the transverse spin of the projectile traveling in the $x^{3}=z$ direction, we define the axis of spin quantization to be the $x^{1}=x$ axis, and we need to construct the Dirac spinors corresponding to the spin projection eigenvalues $\chi= \pm 1$ on the $x$-axis. The proper Lorentz-covariant operator describing the spin orientation is the Pauli-Lubanski vector

$$
W_{\mu} \equiv-\frac{1}{2} \epsilon_{\mu \nu \rho \sigma} S^{\nu \rho} p^{\sigma}
$$

where

$$
S^{\nu \rho} \equiv \frac{i}{4}\left[\gamma^{\nu}, \gamma^{\rho}\right]
$$

are the generators of the Lorentz group and we are using the convention in which $\epsilon_{0123}=+1$.

For our purposes, we need to find the eigenspinors of $W_{1}$ for a particle moving along the $z$-axis. Diagonalizing $W_{1}$ in terms of the pure helicity eigenspinors gives (cf. e.g. [79])

$$
U_{\chi} \equiv \frac{1}{\sqrt{2}}\left[U_{(+z)}+\chi U_{(-z)}\right]
$$


where $U_{ \pm z}$ are the spinors in the helicity basis and $\chi= \pm 1$. These particular spinors $U_{\chi}$ are simultaneous eigenstates of $W_{1}$ and of the Dirac operator:

$$
\begin{gathered}
W_{1} U_{\chi}=\chi \frac{m}{2} U_{\chi} \\
(\gamma \cdot p-m) U_{\chi}=0 .
\end{gathered}
$$

Since the incoming quark is polarized transversely, it is convenient to use the same spinor basis (4) for the outgoing quark in Fig. 7 as well. Using the standard rules of LCPT [57, 58] and working in the transverse polarization basis (44) for the spinors, we evaluate the light-cone wave function shown in Fig. 7 as

$$
\psi_{\lambda \chi \chi^{\prime}}^{a}(\boldsymbol{k}, \boldsymbol{p}, \alpha)=\frac{g T^{a}}{p^{-}-k^{-}-(p-k)^{-}}\left[\frac{\bar{U}_{\chi^{\prime}}(k)}{\sqrt{k^{+}}} \gamma \cdot \epsilon_{\lambda}^{*} \frac{U_{\chi}(p)}{\sqrt{p^{+}}}\right],
$$

where

$$
\epsilon_{\lambda}^{\mu}=\left(0, \frac{2 \boldsymbol{\epsilon}_{\lambda} \cdot(\boldsymbol{p}-\boldsymbol{k})}{p^{+}-k^{+}}, \boldsymbol{\epsilon}_{\lambda}\right)
$$

is the gluon polarization vector with the transverse components $\boldsymbol{\epsilon}_{\lambda}=(-1 / \sqrt{2})(\lambda, i)$.

In arriving at Eq. (5) we have used the fact that the incoming state in Fig. 7 contains only the quark with momentum $p$ while the intermediate state contains the quark and the gluon, as denoted by the vertical dotted line in Fig. 7. For diagrams where the polarized quark scatters in the nucleus before the gluon emission, as shown in the right panel of Fig. 6, the roles are reversed: the quark line $p$ is the intermediate state, and the quark-gluon system is the final state. Since $\sum_{\text {init }} p_{i}^{-}=\sum_{\text {final }} p_{i}^{-}$, the energy denominator reverses sign for final-state splittings.

Using the on-shell conditions explicitly gives the terms entering the energy denominator:

$$
p^{-}=\frac{p_{T}^{2}+m^{2}}{p^{+}}, k^{-}=\frac{k_{T}^{2}+m^{2}}{k^{+}},(p-k)^{-}=\frac{(\boldsymbol{p}-\boldsymbol{k})^{2}}{p^{+}-k^{+}} .
$$

We use the spinors in the Brodsky-Lepage convention [57, [58], which, for a particle moving along the $z$-axis, become helicity eigenstates. The spinor matrix elements for the Brodsky-Lepage spinors are well known [57, 58]. Making the change of basis (4) gives the relevant spinor products as 1

$$
\begin{aligned}
\frac{\bar{U}_{\chi^{\prime}}(k)}{\sqrt{k^{+}}} \gamma^{+} \frac{U_{\chi}(p)}{\sqrt{p^{+}}} & =2 \delta_{\chi, \chi^{\prime}} \\
\frac{\bar{U}_{\chi^{\prime}}(k)}{\sqrt{k^{+}}} \gamma_{\perp}^{i} \frac{U_{\chi}(p)}{\sqrt{p^{+}}} & =\frac{\delta_{\chi, \chi^{\prime}}}{\alpha p^{+}}\left[\left(k_{\perp}^{i}+\alpha p_{\perp}^{i}\right)+(1-\alpha) i m \chi \delta^{i 2}\right] \\
& -\frac{\delta_{\chi,-\chi^{\prime}}}{\alpha p^{+}}\left[i \epsilon^{i j}\left(k_{\perp}^{j}-\alpha p_{\perp}^{j}\right)+(1-\alpha) m \chi \delta^{i 1}\right],
\end{aligned}
$$

and the $\gamma^{-}$matrix element does not contribute to $\gamma \cdot \epsilon_{\lambda}^{*}$ since $\epsilon_{\lambda}^{+}=0$ in the light-cone gauge. Here $\epsilon^{12}=-\epsilon^{21}=1$, $\epsilon^{11}=\epsilon^{22}=0$. With the matrix elements (8) it is straightforward to evaluate the light-cone wave function (5) in momentum space, obtaining

$$
\begin{aligned}
\psi_{\lambda \chi \chi^{\prime}}^{a}(\boldsymbol{k}, \boldsymbol{p}, \alpha) & =\frac{g T^{a}}{(\boldsymbol{k}-\alpha \boldsymbol{p})^{2}+\tilde{m}^{2}} \\
& \times\left[\boldsymbol{\epsilon}_{\lambda}^{*} \cdot(\boldsymbol{k}-\alpha \boldsymbol{p})\left((1+\alpha) \delta_{\chi \chi^{\prime}}+\lambda(1-\alpha) \delta_{\chi,-\chi^{\prime}}\right)-\frac{\tilde{m}}{\sqrt{2}}(1-\alpha) \chi\left(\delta_{\chi \chi^{\prime}}-\lambda \delta_{\chi,-\chi^{\prime}}\right)\right],
\end{aligned}
$$

where

$$
\tilde{m} \equiv(1-\alpha) m
$$

is a natural effective mass parameter in the wave function and $T^{a}$ are the $\mathrm{SU}\left(N_{c}\right)$ generators in the fundamental representation.

\footnotetext{
${ }^{1}$ Note again that $U_{\chi}(p)$ becomes a spinor for a transversely polarized particle only for $\boldsymbol{p}=0$ : Eqs. 8) give us the matrix elements for spinors related to the Brodsky-Lepage spinors via Eq. (4), which do not necessarily correspond to transverse polarizations in the general case.
} 
Now we can Fourier transform the wave function to coordinate space

$$
\psi_{\lambda \chi \chi^{\prime}}^{a}(\boldsymbol{x}, \boldsymbol{z}, \alpha ; \boldsymbol{u}) \equiv \int \frac{d^{2} k}{(2 \pi)^{2}} \frac{d^{2} p}{(2 \pi)^{2}} e^{i \boldsymbol{k} \cdot(\boldsymbol{z}-\boldsymbol{x})} e^{i \boldsymbol{p} \cdot(\boldsymbol{x}-\boldsymbol{u})} \psi_{\lambda \chi \chi^{\prime}}^{a}(\boldsymbol{k}, \boldsymbol{p}, \alpha)
$$

with the transverse coordinates defined in Fig. 7 Since the momentum-space wave function depends only on $\boldsymbol{k}-\alpha \boldsymbol{p}$, one of the two integrals can be performed to yield a delta function $\delta^{2}[(\boldsymbol{x}-\boldsymbol{u})+\alpha(\boldsymbol{z}-\boldsymbol{x})]$. Performing the remaining momentum integral in Eq. (10) yields

$$
\begin{aligned}
\psi_{\lambda \chi \chi^{\prime}}^{a}(\boldsymbol{x}, \boldsymbol{z}, \alpha ; \boldsymbol{u}) & =\frac{g T^{a}}{2 \pi} \delta^{2}[(\boldsymbol{x}-\boldsymbol{u})+\alpha(\boldsymbol{z}-\boldsymbol{x})] \tilde{m}\left\{i \boldsymbol{\epsilon}_{\lambda}^{*} \cdot \frac{\boldsymbol{z}-\boldsymbol{x}}{|\boldsymbol{z}-\boldsymbol{x}|} K_{1}(\tilde{m}|\boldsymbol{z}-\boldsymbol{x}|)\left[(1+\alpha) \delta_{\chi, \chi^{\prime}}+\lambda(1-\alpha) \delta_{\chi,-\chi^{\prime}}\right]\right. \\
& \left.-\frac{\chi(1-\alpha)}{\sqrt{2}} K_{0}(\tilde{m}|\boldsymbol{z}-\boldsymbol{x}|)\left[\delta_{\chi, \chi^{\prime}}-\lambda \delta_{\chi,-\chi^{\prime}}\right]\right\}
\end{aligned}
$$

It is useful to separate out the color factor $T^{a}$ and the delta function from the rest of the wave function (denoted by $\left.\Psi_{\lambda \chi \chi^{\prime}}\right)$, such that

$$
\psi_{\lambda \chi \chi^{\prime}}^{a}(\boldsymbol{x}, \boldsymbol{z}, \alpha ; \boldsymbol{u}) \equiv T^{a} \delta^{2}[(\boldsymbol{u}-\boldsymbol{x})-\alpha(\boldsymbol{z}-\boldsymbol{x})] \Psi_{\lambda \chi \chi^{\prime}}(\boldsymbol{z}-\boldsymbol{x}, \alpha)
$$

Finally, we need to square the wave function and sum over the final particles' polarizations. Here we are interested in producing a quark with a fixed transverse momentum, while integrating over all transverse momenta of the produced gluon in Fig. 6] According to the standard prescription [56, 76], for coordinate-space scattering amplitudes this means that the gluon's transverse coordinate $\boldsymbol{x}$ will be the same both in the amplitude and in the complex conjugate amplitude (since its transverse momentum is integrated over in the cross section), while the quarks have different transverse coordinates between the amplitude and the conjugate amplitude (since they are the observed particles). (See Fig. 10 below for the illustration of the amplitude squared.) The "square" of the light cone wave function (11) with the above rule for the quark and gluon transverse coordinates is illustrated in Fig. 8 .

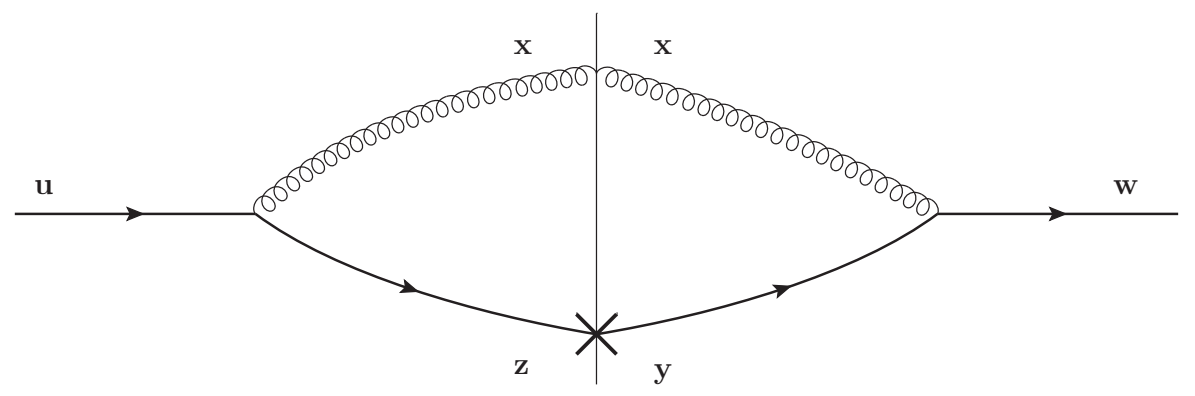

FIG. 8. Light-cone wave function from Fig. 7 squared. The vertical straight line separates the wave function from its conjugate, while the cross denotes the quark that we tag on. The untagged gluon's coordinate $\boldsymbol{x}$ is unchanged, but the quark coordinates differ ( $\boldsymbol{z}$ and $\boldsymbol{u}$ vs. $\boldsymbol{y}$ and $\boldsymbol{w}$, as explained in the text.)

The wave function (11) squared $\Phi_{\chi}$ as shown in Fig. 8 contains one contribution which is polarization-independent and another which is proportional to the quark polarization eigenvalue $\chi$

$$
\Phi_{\chi}(\boldsymbol{z}-\boldsymbol{x}, \boldsymbol{y}-\boldsymbol{x}, \alpha) \equiv \sum_{\lambda, \chi^{\prime}= \pm 1} \Psi_{\lambda \chi \chi^{\prime}}(\boldsymbol{z}-\boldsymbol{x}, \alpha) \Psi_{\lambda \chi \chi^{\prime}}^{*}(\boldsymbol{y}-\boldsymbol{x}, \alpha) \equiv \Phi_{\text {unp }}(\boldsymbol{z}-\boldsymbol{x}, \boldsymbol{y}-\boldsymbol{x}, \alpha)+\chi \Phi_{p o l}(\boldsymbol{z}-\boldsymbol{x}, \boldsymbol{y}-\boldsymbol{x}, \alpha)
$$

Substituting the wave function (11) into Eq. (13) and performing the sums gives the unpolarized part as

$$
\begin{aligned}
\Phi_{u n p}= & \frac{2 \alpha_{s}}{\pi} \tilde{m}^{2}\left[\left(1+\alpha^{2}\right) \frac{(\boldsymbol{z}-\boldsymbol{x}) \cdot(\boldsymbol{y}-\boldsymbol{x})}{|\boldsymbol{z}-\boldsymbol{x}||\boldsymbol{y}-\boldsymbol{x}|} K_{1}(\tilde{m}|\boldsymbol{z}-\boldsymbol{x}|) K_{1}(\tilde{m}|\boldsymbol{y}-\boldsymbol{x}|)\right. \\
& \left.+(1-\alpha)^{2} K_{0}(\tilde{m}|\boldsymbol{z}-\boldsymbol{x}|) K_{0}(\tilde{m}|\boldsymbol{y}-\boldsymbol{x}|)\right]
\end{aligned}
$$

and the transversely-polarized part as

$$
\Phi_{\text {pol }}=\frac{2 \alpha_{s}}{\pi} \tilde{m}^{2} \alpha(1-\alpha)\left[\frac{z^{2}-x^{2}}{|\boldsymbol{z}-\boldsymbol{x}|} K_{0}(\tilde{m}|\boldsymbol{y}-\boldsymbol{x}|) K_{1}(\tilde{m}|\boldsymbol{z}-\boldsymbol{x}|)+\frac{y^{2}-x^{2}}{|\boldsymbol{y}-\boldsymbol{x}|} K_{1}(\tilde{m}|\boldsymbol{y}-\boldsymbol{x}|) K_{0}(\tilde{m}|\boldsymbol{z}-\boldsymbol{x}|)\right]
$$


Note that $\Phi_{\text {unp }}$ is a scalar under rotations in the transverse plane, whereas $\Phi_{\text {pol }}$ has an explicitly preferred $x^{2}$-direction (i.e., the y-axis) since it "knows" about the transverse polarization of the incoming quark. The $x^{2}$-axis can be written as the direction of the $\vec{p} \times \vec{S}$ vector, since the incoming quark with momentum $\vec{p}$ is moving along the $z$-axis, while being polarized along the $x=x^{1}$-axis, such that $\vec{S} \| \hat{x}_{1}$. We show that the unpolarized part of the wave function squared $\Phi_{\text {unp }}$ contributes to the unpolarized quark production cross section $d \sigma_{\text {unp }}$, while the polarization-dependent part of the wave function squared $\Phi_{\text {pol }}$ generates the spin-asymmetric cross section $d(\Delta \sigma)$.

\section{B. Spin, Asymmetry, and C-Parity in Quark Production}

Having computed the $q \rightarrow q G$ light-cone wave function, we can now construct the scattering cross section by allowing the wave function to interact with the small- $x$ field of the target nucleus. It is well known [44, 49] that eikonal quark and gluon propagators in the background color field $A^{\mu a}$ can be correspondingly written as fundamental and adjoint path-ordered Wilson lines

$$
\begin{aligned}
V_{\boldsymbol{x}} & \equiv \mathcal{P} \exp \left[\frac{i g}{2} \int_{-\infty}^{+\infty} d x^{+} T^{a} A^{-a}\left(x^{+}, x^{-}=0, \boldsymbol{x}\right)\right] \\
U_{\boldsymbol{x}}^{b a} & \equiv \mathcal{P} \exp \left[\frac{i g}{2} \int_{-\infty}^{+\infty} d x^{+} t^{c} A^{-c}\left(x^{+}, x^{-}=0, \boldsymbol{x}\right)\right]^{b a},
\end{aligned}
$$

where $t^{a}$ 's are the $\mathrm{SU}\left(N_{c}\right)$ generators in the adjoint representation and the projectile is moving along the light-cone $x^{+}$-axis. In essence, this means that the the projectile's transverse position is not altered during the scattering, and the effect of the target field is to perform a net $\mathrm{SU}\left(N_{c}\right)$ color rotation on the projectile. The Wilson lines resum these interactions and give the total phase of that color rotation. They are illustrated in Fig. 9. Note that the adjoint Wilson line $U_{\boldsymbol{x}}^{b a}$ is real-valued.
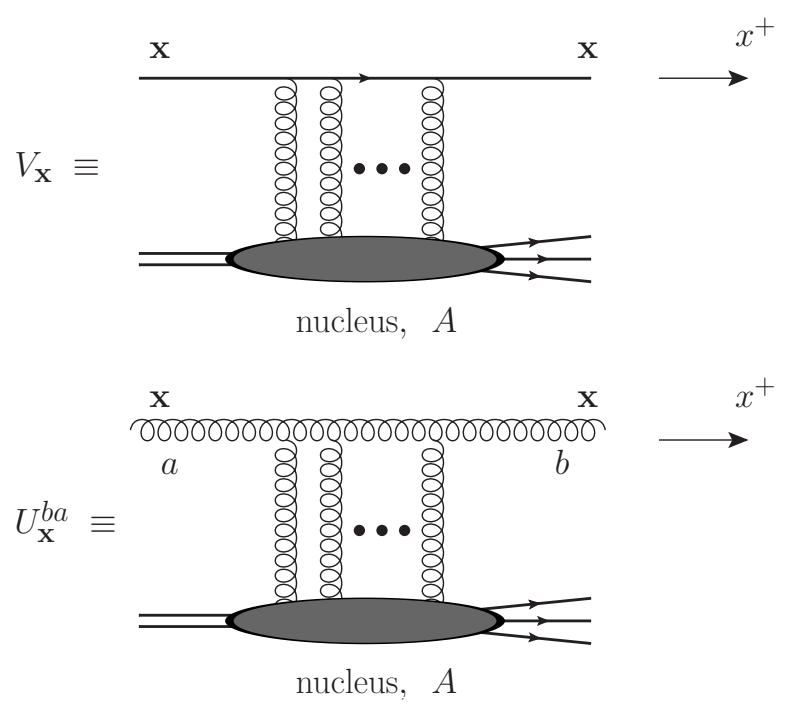

FIG. 9. Wilson lines resumming scattering in the small- $x$ field of the target. The quark propagator is in the fundamental representation (top), and the gluon propagator is in the adjoint representation (bottom).

The Wilson-line approach is quite generic: if the target gluon field is quasi-classical, as in the case of the McLerranVenugopalan (MV) model [35 37], then correlators of the Wilson lines resum powers of $\alpha_{s}^{2} A^{1 / 3}$ corresponding to the Glauber-Mueller multiple-rescattering approximation [75]. Non-linear small- $x$ evolution resumming powers of $\alpha_{s} Y \sim \alpha_{s} \ln s$ can be included into the correlators of the Wilson lines through the Balitsky-Kovchegov (BK) [4953] and Jalilian-Marian-Iancu-McLerran-Weigert-Leonidov-Kovner (JIMWLK) 40 48] evolution equations. Thus expressing the interaction with the target in terms of the Wilson lines (16) allows for several different levels of approximation for this interaction.

The scattering amplitude for quark production is composed of two sub-processes: the splitting of Eq. (11) calculated in Sec. II A and the Wilson line scattering (16) of the quark and the gluon in the field of the target. These elements give 

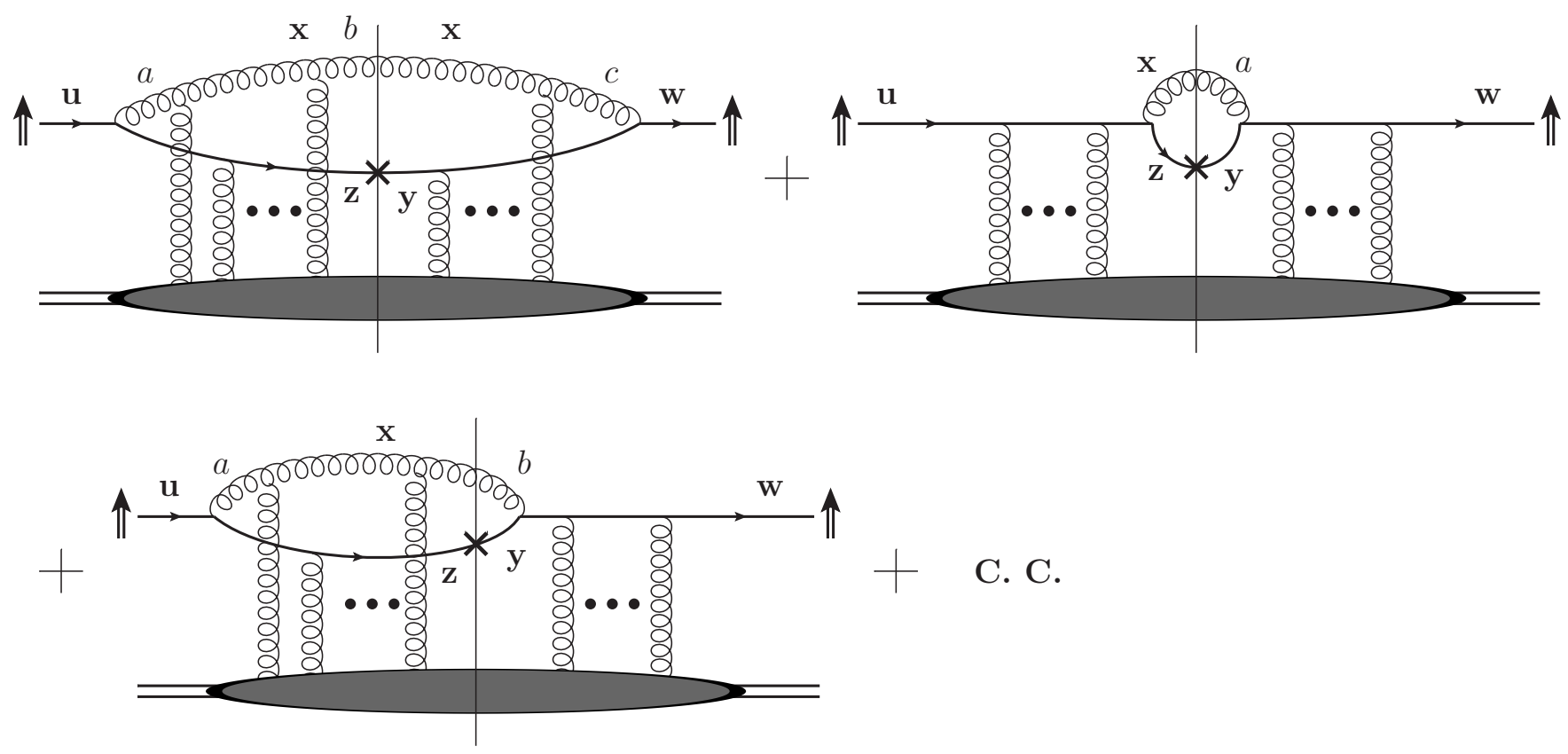

FIG. 10. The cross section for quark production in the polarized quark-nucleus scattering.

two distinct diagrams contributing to the scattering amplitude shown in Fig. 6 above. To find the quark production cross section we need to square the diagrams in Fig. 6. keeping the transverse momentum of the quark fixed, as depicted in Fig. 10, As discussed above, this implies that the transverse coordinates of the quark are different on both sides of the cut. Just like in other similar calculations [76, 78] the $q \rightarrow q G$ splitting may occur either before or after the interaction with the target, both in the amplitude and in the complex conjugate amplitude, resulting in the four different terms shown in Fig. 10.

Using Fig. 10 we can write down the expression for the color-averaged amplitude squared $\left\langle M^{2}\right\rangle$ in terms of Wilson lines and the wave function responsible for the splitting, remembering to reverse the sign in the wave function for splitting occurring after the interaction:

$$
\begin{aligned}
\left\langle M^{2}\right\rangle & =\frac{1}{N_{c}} \sum_{\lambda, \chi^{\prime}}\left[\operatorname{Tr}\left[V_{\boldsymbol{z}} \psi_{\lambda \chi \chi^{\prime}}^{a} \psi_{\lambda \chi \chi^{\prime}}^{c \dagger} V_{\boldsymbol{y}}^{\dagger}\right] U_{\boldsymbol{x}}^{b a} U_{\boldsymbol{x}}^{b c}+\operatorname{Tr}\left[\psi_{\lambda \chi \chi^{\prime}}^{a}, V_{\boldsymbol{u}} V_{\boldsymbol{w}}^{\dagger} \psi_{\lambda \chi \chi^{\prime}}^{a \dagger}\right]\right. \\
& \left.-\operatorname{Tr}\left[V_{\boldsymbol{z}} \psi_{\lambda \chi \chi^{\prime}}^{a} V_{\boldsymbol{w}}^{\dagger} \psi_{\lambda \chi \chi^{\prime}}^{b \dagger}\right] U_{\boldsymbol{x}}^{b a}-\operatorname{Tr}\left[\psi_{\lambda \chi \chi^{\prime}}^{a}, V_{\boldsymbol{u}} \psi_{\lambda \chi \chi^{\prime}}^{b \dagger} V_{\boldsymbol{y}}^{\dagger}\right] U_{\boldsymbol{x}}^{a b}\right]
\end{aligned}
$$

where $N_{c}$ is the number of colors, the traces are taken over the fundamental representation indices, and summation is implied over repeated adjoint color indices. Substituting Eq. (12) into Eq. (17) and using the identities

$$
U_{\boldsymbol{x}}^{b a} T^{a}=V_{\boldsymbol{x}}^{\dagger} T^{b} V_{\boldsymbol{x}} \quad, \quad \operatorname{Tr}\left[A T^{a} B T^{a}\right]=\frac{1}{2} \operatorname{Tr} A \operatorname{Tr} B-\frac{1}{2 N_{c}} \operatorname{Tr}[A B]
$$

for arbitrary $N_{c} \times N_{c}$ matrices $A, B$, we find

$$
\left\langle M^{2}\right\rangle=C_{F} \delta^{2}[\boldsymbol{u}-\boldsymbol{x}-\alpha(\boldsymbol{z}-\boldsymbol{x})] \delta^{2}[\boldsymbol{w}-\boldsymbol{x}-\alpha(\boldsymbol{y}-\boldsymbol{x})] \Phi_{\chi}(\boldsymbol{z}-\boldsymbol{x}, \boldsymbol{y}-\boldsymbol{x}) \mathcal{I}^{(q)}
$$

where the factor responsible for the quark's interaction with the target, denoted by $\mathcal{I}^{(q)}$, is given by

$$
\begin{aligned}
\mathcal{I}^{(q)}=\left\langle\frac{1}{N_{c}} \operatorname{Tr}\left[V_{\boldsymbol{z}} V_{\boldsymbol{y}}^{\dagger}\right]+\frac{1}{N_{c}} \operatorname{Tr}\left[V_{\boldsymbol{u}} V_{\boldsymbol{w}}^{\dagger}\right]\right. & -\frac{1}{2 N_{c} C_{F}} \operatorname{Tr}\left[V_{\boldsymbol{z}} V_{\boldsymbol{x}}^{\dagger}\right] \operatorname{Tr}\left[V_{\boldsymbol{x}} V_{\boldsymbol{w}}^{\dagger}\right]+\frac{1}{2 N_{c}^{2} C_{F}} \operatorname{Tr}\left[V_{\boldsymbol{z}} V_{\boldsymbol{w}}^{\dagger}\right] \\
& \left.-\frac{1}{2 N_{c} C_{F}} \operatorname{Tr}\left[V_{\boldsymbol{u}} V_{\boldsymbol{x}}^{\dagger}\right] \operatorname{Tr}\left[V_{\boldsymbol{x}} V_{\boldsymbol{y}}^{\dagger}\right]+\frac{1}{2 N_{c}^{2} C_{F}} \operatorname{Tr}\left[V_{\boldsymbol{u}} V_{\boldsymbol{y}}^{\dagger}\right]\right\rangle .
\end{aligned}
$$

Here $C_{F}=\left(N_{c}^{2}-1\right) / 2 N_{c}$ is the fundamental Casimir operator of $\mathrm{SU}\left(N_{c}\right)$, and the angle brackets on the right denote averaging over the field configurations of the target. 
Defining the $S$-matrix operator for a fundamental-representation color dipole by

$$
\hat{D}_{\boldsymbol{x} \boldsymbol{y}} \equiv \frac{1}{N_{c}} \operatorname{Tr}\left[V_{\boldsymbol{x}} V_{\boldsymbol{y}}^{\dagger}\right]
$$

we can rewrite $\mathcal{I}^{(q)}$ more compactly as

$$
\mathcal{I}^{(q)}=\left\langle\hat{D}_{\boldsymbol{z} \boldsymbol{y}}+\hat{D}_{\boldsymbol{u} \boldsymbol{w}}-\frac{N_{c}}{2 C_{F}} \hat{D}_{\boldsymbol{z} \boldsymbol{x}} \hat{D}_{\boldsymbol{x} \boldsymbol{w}}+\frac{1}{2 N_{c} C_{F}} \hat{D}_{\boldsymbol{z} \boldsymbol{w}}-\frac{N_{c}}{2 C_{F}} \hat{D}_{\boldsymbol{u} \boldsymbol{x}} \hat{D}_{\boldsymbol{x} \boldsymbol{y}}+\frac{1}{2 N_{c} C_{F}} \hat{D}_{\boldsymbol{u} \boldsymbol{y}}\right\rangle .
$$

As we have already mentioned, this interaction with the target can be evaluated either in the Glauber-Mueller multiple-rescattering approximation or using the JIMWLK evolution equation.

The expression (22) simplifies in 't Hooft's large- $N_{c}$ limit, in which the correlators of several single-trace operators factorize, such that, for instance, $\left\langle\hat{D}_{\boldsymbol{u} \boldsymbol{x}} \hat{D}_{\boldsymbol{x} \boldsymbol{y}}\right\rangle=\left\langle\hat{D}_{\boldsymbol{u} \boldsymbol{x}}\right\rangle\left\langle\hat{D}_{\boldsymbol{x} \boldsymbol{y}}\right\rangle$ [49, 52, 55]. Defining

$$
D_{\boldsymbol{x} \boldsymbol{y}} \equiv\left\langle\hat{D}_{\boldsymbol{x} \boldsymbol{y}}\right\rangle=\frac{1}{N_{c}}\left\langle\operatorname{Tr}\left[V_{\boldsymbol{x}} V_{\boldsymbol{y}}^{\dagger}\right]\right\rangle
$$

we rewrite Eq. (22) in the large- $N_{c}$ limit as

$$
\left.\mathcal{I}^{(q)}\right|_{\text {large }-N_{c}}=D_{\boldsymbol{z} \boldsymbol{y}}+D_{\boldsymbol{u} \boldsymbol{w}}-D_{\boldsymbol{z} \boldsymbol{x}} D_{\boldsymbol{x} \boldsymbol{w}}-D_{\boldsymbol{u} \boldsymbol{x}} D_{\boldsymbol{x} \boldsymbol{y}}
$$

To compute the quark production cross sections, we need to Fourier transform the coordinate space amplitude squared of Eq. (19) back to momentum space and include the appropriate kinematic factors. This is accomplished by [56]

$$
\frac{d \sigma^{(q)}}{d^{2} k d y_{q}}=\frac{1}{2(2 \pi)^{3}} \frac{\alpha}{1-\alpha} \int d^{2} x d^{2} y d^{2} z d^{2} u d^{2} w e^{-i \boldsymbol{k} \cdot(\boldsymbol{z}-\boldsymbol{y})} e^{i \boldsymbol{p} \cdot(\boldsymbol{u}-\boldsymbol{w})}\left\langle M^{2}\right\rangle
$$

with $\boldsymbol{k}$ and $y_{q}$ the transverse momentum and rapidity of the produced quark. Integrating over the delta functions in (19) imposes the kinematic constraints

$$
\begin{aligned}
\boldsymbol{u} & =\boldsymbol{x}+\alpha(\boldsymbol{z}-\boldsymbol{x}) \\
\boldsymbol{w} & =\boldsymbol{x}+\alpha(\boldsymbol{y}-\boldsymbol{x})
\end{aligned}
$$

which relate the quark coordinates before and after the $q \rightarrow q G$ splitting and describe the non-eikonal quark recoil. To make the incoming quark transversely polarized we need to put the transverse momentum of the incoming quark to zero: $\boldsymbol{p}=\mathbf{0}$. We thus obtain the general result for the quark production in the $q^{\uparrow}+A$ scattering

$$
\frac{d \sigma^{(q)}}{d^{2} k d y_{q}}=\frac{C_{F}}{2(2 \pi)^{3}} \frac{\alpha}{1-\alpha} \int d^{2} x d^{2} y d^{2} z e^{-i \boldsymbol{k} \cdot(\boldsymbol{z}-\boldsymbol{y})} \Phi_{\chi}(\boldsymbol{z}-\boldsymbol{x}, \boldsymbol{y}-\boldsymbol{x}, \alpha) \mathcal{I}^{(q)}(\boldsymbol{x}, \boldsymbol{y}, \boldsymbol{z})
$$

with $\Phi_{\chi}$ from Eq. (13) and $\mathcal{I}^{(q)}$ from Eq. (22). The expression (27) contains multiple rescatterings and non-linear small- $x$ evolution between the projectile and the target. Note that it does not resum the small- $x$ evolution between the produced quark and the projectile (which can be included following [78]), and hence is not valid for very small $\alpha$ (i.e., the values of $\alpha$ are restricted by $\alpha_{s} \ln 1 / \alpha \ll 1$ ). Since, as we will see below, both the experimental STSA and STSA resulting from our production mechanism fall off with decreasing $\alpha$, the region of interest in this work corresponds to $\alpha$ not being very small, where Eq. (27) is fully applicable.

Now we are in a position to analyze the symmetry properties of the wave function and the interaction. There are two relevant symmetries to consider: $\boldsymbol{k} \rightarrow-\boldsymbol{k}$ or " $k_{T}$-parity" and $\chi \rightarrow-\chi$ spin-flip. Under $k_{T}$-parity, the quark and antiquark coordinates $\boldsymbol{z}$ and $\boldsymbol{y}$ get interchanged, $\boldsymbol{z} \leftrightarrow \boldsymbol{y}$. (Note that, due to Eqs. (26), this also implies that $\boldsymbol{u} \leftrightarrow \boldsymbol{w}$.) From our previous calculation of the wave function (14), (15), we note that $\Phi_{\text {unp }}$ is spin-independent, a scalar under rotations in the transverse plane, and $k_{T}$-even. Similarly, $\chi \Phi_{\text {pol }}$ is odd under spin-flip, a vector under transverse rotations, and $k_{T}$-even. We can explicitly (anti-)symmetrize the interaction with the target under $\boldsymbol{z} \leftrightarrow \boldsymbol{y}$ interchange

$$
\mathcal{I}_{\text {symm / anti }} \equiv \frac{1}{2}(\mathcal{I} \pm(\boldsymbol{z} \leftrightarrow \boldsymbol{y}))
$$


To do so we first need to decompose each dipole $S$-matrix into the even and odd pieces under the exchange of transverse coordinates, which corresponds to the $C$-parity operation exchanging the quark and the anti-quark [71, 72]:

$$
\begin{aligned}
\hat{D}_{\boldsymbol{x} \boldsymbol{y}} & \equiv \hat{S}_{\boldsymbol{x} \boldsymbol{y}}+i \hat{O}_{\boldsymbol{x} \boldsymbol{y}} \\
\hat{S}_{\boldsymbol{x} \boldsymbol{y}} & \equiv \frac{1}{2}\left(\hat{D}_{\boldsymbol{x} \boldsymbol{y}}+\hat{D}_{\boldsymbol{y} \boldsymbol{x}}\right) \\
\hat{O}_{\boldsymbol{x} \boldsymbol{y}} & \equiv \frac{1}{2 i}\left(\hat{D}_{\boldsymbol{x} \boldsymbol{y}}-\hat{D}_{\boldsymbol{y} \boldsymbol{x}}\right) .
\end{aligned}
$$

The $C$-even real part of the target field-averaged $S$-matrix $S_{\boldsymbol{x} \boldsymbol{y}} \equiv\left\langle\hat{S}_{\boldsymbol{x} \boldsymbol{y}}\right\rangle$ is responsible for the total unpolarized cross section of the dipole-target interactions. Its small- $x$ evolution is given by the BK/JIMWLK equations. The $C$-odd imaginary part of the target-averaged $S$-matrix $O_{x y} \equiv\left\langle\hat{O}_{x \boldsymbol{u}}\right\rangle$ is known as the odderon interaction [67 69]. The small- $x$ evolution equation for $O_{x y}$ was constructed in [71 73], and, in the linear approximation, was found to be identical to the dipole BFKL equation [32] with $C$-odd initial conditions. The intercept of the linearized odderon evolution was found to be $\alpha_{O}-1=0$, in agreement with the solution of the Bartels-Kwiecinski-Praszalowicz (BKP) [80, 81] equation for the odderon found in 70]. For the current status of the experimental searches for the QCD odderon and for an overview of the theory see [69].

With these explicitly symmetrized elements, it is straightforward to construct the symmetric and antisymmetric parts of the interaction with the target (22) for quark production:

$$
\begin{aligned}
\mathcal{I}_{s y m m}^{(q)}= & \left\langle\hat{S}_{\boldsymbol{z} \boldsymbol{y}}+\hat{S}_{\boldsymbol{u} \boldsymbol{w}}-\frac{N_{c}}{2 C_{F}}\left(\hat{S}_{\boldsymbol{z} \boldsymbol{x}} \hat{S}_{\boldsymbol{x} \boldsymbol{w}}-\hat{O}_{\boldsymbol{z} \boldsymbol{x}} \hat{O}_{\boldsymbol{x} \boldsymbol{w}}\right)+\frac{1}{2 N_{c} C_{F}} \hat{S}_{\boldsymbol{z} \boldsymbol{w}}\right. \\
& \left.-\frac{N_{c}}{2 C_{F}}\left(\hat{S}_{\boldsymbol{u} \boldsymbol{x}} \hat{S}_{\boldsymbol{x} \boldsymbol{y}}-\hat{O}_{\boldsymbol{u} \boldsymbol{x}} \hat{O}_{\boldsymbol{x} \boldsymbol{y}}\right)+\frac{1}{2 N_{c} C_{F}} \hat{S}_{\boldsymbol{u} \boldsymbol{y}}\right\rangle \\
\mathcal{I}_{\text {anti }}^{(q)}= & i\left\langle\hat{O}_{\boldsymbol{z} \boldsymbol{y}}+\hat{O}_{\boldsymbol{u} \boldsymbol{w}}-\frac{N_{c}}{2 C_{F}}\left(\hat{O}_{\boldsymbol{z} \boldsymbol{x}} \hat{S}_{\boldsymbol{x} \boldsymbol{w}}+\hat{S}_{\boldsymbol{z} \boldsymbol{x}} \hat{O}_{\boldsymbol{x} \boldsymbol{w}}\right)+\frac{1}{2 N_{c} C_{F}} \hat{O}_{\boldsymbol{z} \boldsymbol{w}}\right. \\
& \left.-\frac{N_{c}}{2 C_{F}}\left(\hat{O}_{\boldsymbol{u} \boldsymbol{x}} \hat{S}_{\boldsymbol{x} \boldsymbol{y}}+\hat{S}_{\boldsymbol{u} \boldsymbol{x}} \hat{O}_{\boldsymbol{x} \boldsymbol{y}}\right)+\frac{1}{2 N_{c} C_{F}} \hat{O}_{\boldsymbol{u} \boldsymbol{y}}\right\rangle .
\end{aligned}
$$

In the large- $N_{c}$ limit these expressions simplify to

$$
\begin{gathered}
\left.\mathcal{I}_{s y m m}^{(q)}\right|_{\operatorname{large}-N_{c}}=S_{\boldsymbol{z} \boldsymbol{y}}+S_{\boldsymbol{u} \boldsymbol{w}}-S_{\boldsymbol{z} \boldsymbol{x}} S_{\boldsymbol{x} \boldsymbol{w}}-S_{\boldsymbol{u} \boldsymbol{x}} S_{\boldsymbol{x} \boldsymbol{y}}+O_{\boldsymbol{z} \boldsymbol{x}} O_{\boldsymbol{x} \boldsymbol{w}}+O_{\boldsymbol{u} \boldsymbol{x}} O_{\boldsymbol{x} \boldsymbol{y}}, \\
\mathcal{I}_{\text {anti }\left.i\right|_{\operatorname{large}-N_{c}} ^{(q)}}=i\left[O_{\boldsymbol{z} \boldsymbol{y}}+O_{\boldsymbol{u} \boldsymbol{w}}-O_{\boldsymbol{z} \boldsymbol{x}} S_{\boldsymbol{x} \boldsymbol{w}}-O_{\boldsymbol{u} \boldsymbol{x}} S_{\boldsymbol{x} \boldsymbol{y}}-S_{\boldsymbol{z} \boldsymbol{x}} O_{\boldsymbol{x} \boldsymbol{w}}-S_{\boldsymbol{u} \boldsymbol{x}} O_{\boldsymbol{x} \boldsymbol{y}}\right] .
\end{gathered}
$$

Because the production cross section preserves azimuthal rotational symmetry, any physical observable must behave the same way under both $k_{T}$-parity and spin flip; that is, the only terms which are non-zero are those which are even under the combined operation $k_{T} \otimes$ (spin flip). Note that $\Phi_{u n p}$ is even under the spin-flip, while $\chi \Phi_{\text {pol }}$ is odd under spin-flip, and both are even under $k_{T}$-parity. Hence in the $\Phi_{\chi} \mathcal{I}^{(q)}$ product in Eq. (27) the terms which give non-zero contributions to the cross section are $\Phi_{\text {unp }} \mathcal{I}_{\text {symm }}^{(q)}$ and $\chi \Phi_{\text {pol }} \mathcal{I}_{\text {anti }}^{(q)}$. The other terms $\Phi_{\text {unp }} \mathcal{I}_{\text {anti }}^{(q)}$ and $\chi \Phi_{\text {pol }} \mathcal{I}_{\text {symm }}^{(q)}$ contain a contradiction between their vector structure and their $k_{T}$-parity, so they vanish identically: for instance, the contribution to the cross section coming from $\Phi_{u n p} \mathcal{I}_{\text {anti }}^{(q)}$ is odd under $k_{T}$-parity and is a function of $k_{T}^{2}$ only, which is possible only if it is zero. Similarly, the contribution of $\chi \Phi_{p o l} \mathcal{I}_{s y m m}^{(q)}$ is even under $k_{T}$-parity and is a vector in transverse space, which implies that it is also zero. Of the nonzero terms, $\Phi_{\text {unp }} \mathcal{I}_{s y m m}^{(q)}$ does not change its sign under spin-flip; it generates the symmetric part of the distribution $d \sigma_{\text {unp }}$ in Eq. (11). At the same time $\chi \Phi_{\text {pol }} \mathcal{I}_{\text {anti }}^{(q)}$ does change sign under spin-flip; it generates the transverse spin asymmetry we are looking for.

Knowing these symmetry properties, we can explicitly construct the spin-dependent and spin-averaged cross sections $d(\Delta \sigma)$ and $d \sigma_{u n p}$ for quark production from their definitions (1) obtaining

$$
\begin{aligned}
d\left(\Delta \sigma^{(q)}\right) & =\frac{C_{F}}{(2 \pi)^{3}} \frac{\alpha}{1-\alpha} \int d^{2} x d^{2} y d^{2} z e^{-i \boldsymbol{k} \cdot(\boldsymbol{z}-\boldsymbol{y})} \Phi_{p o l}(\boldsymbol{z}-\boldsymbol{x}, \boldsymbol{y}-\boldsymbol{x}, \alpha) \mathcal{I}_{\text {anti }}^{(q)}(\boldsymbol{x}, \boldsymbol{y}, \boldsymbol{z}) \\
d \sigma_{u n p}^{(q)} & =\frac{C_{F}}{2(2 \pi)^{3}} \frac{\alpha}{1-\alpha} \int d^{2} x d^{2} y d^{2} z e^{-i \boldsymbol{k} \cdot(\boldsymbol{z}-\boldsymbol{y})} \Phi_{u n p}(\boldsymbol{z}-\boldsymbol{x}, \boldsymbol{y}-\boldsymbol{x}, \alpha) \mathcal{I}_{\text {symm }}^{(q)}(\boldsymbol{x}, \boldsymbol{y}, \boldsymbol{z}),
\end{aligned}
$$

where the wave functions squared are given by Eqs. (14), (15), and the interactions are given by Eqs. (30) (and by Eqs. (31) in the large- $N_{c}$ limit). Eqs. (32) are one of the main results of this work: together with Eq. (10) they give the single-transverse spin asymmetry $A_{N}$ generated in quark production by the $C$-odd CGC interactions with the target. 
The mechanism for the generation of the STSA in Eqs. (32) is different from both the well-known Sivers [15, 16] and Collins [27] effects. It appears difficult (if not impossible) to absorb the interactions of Fig. 10 into the projectile wave function (distribution function): hence our result is different from the Sivers effect. In the above calculation the asymmetry is generated before fragmentation; hence the STSA resulting from Eqs. (32) cannot be due to Collins effect either. As we will see below, the non-zero part of Eq. (32a) stems from the multiple interactions with the target (higher-twist effects), and its contribution is in fact zero in the linearized (leading-twist) approximation. In this sense the above mechanism for generating STSA is similar in spirit to the higher-twist mechanisms of [17 26], though a detailed comparison of the diagrams appears to indicate that the two approaches are, in fact, different.

We have shown explicitly that the single-transverse spin asymmetry $A_{N}$ occurs in the CGC framework as a coupling between the transverse spin of the projectile and a $C$-odd interaction with the target, driven by the odderon 2 Note that to date there is no unambiguous experimental evidence for the QCD odderon. If our mechanism for generating STSA can be isolated experimentally from other contributions, it may constitute the first direct observation of the QCD odderon! To make such a distinction possible, one needs to determine phenomenological characteristics of our mechanism, such as its rapidity, energy, and centrality dependence; some of this work will be carried out below, while the rest, along with a proper phenomenological implementation of our results, is left for future work.

Within LCPT, the real-virtual cancellations or "crossing symmetry" embodied in Eq. (18) allow particles appearing in the complex conjugate amplitude $M^{*}$ to be rewritten as their charge-conjugate particles appearing in the amplitude $M$. This is what gives rise to the natural dipole degrees of freedom within CGC, and in terms of the asymmetry, this feature translates the $k_{T}$-parity of the cross section into the $C$-parity of the interaction with the target. This is the reason the odderon appears naturally in the expression for the asymmetry.

In the literature it is often emphasized that the STSA $A_{N}$ is odd under the time reversal transformation $T$ [27]. To elucidate how the obtained result transforms under $T$ we note that $T$ reverses the directions of $\vec{S}, \vec{p}$, and $\vec{k}$ :

$$
\vec{S} \stackrel{T}{\rightarrow}-\vec{S}, \quad \vec{p} \stackrel{T}{\rightarrow}-\vec{p}, \quad \vec{k} \stackrel{T}{\rightarrow}-\vec{k} .
$$

Since $(\vec{p} \times \vec{S}) \| \hat{x}_{2}$ is invariant under $T$, the only effect of time-reversal on the spin-dependent cross section (32a) is

$$
d\left(\Delta \sigma^{(q)}\right)(\boldsymbol{k}) \stackrel{T}{\rightarrow} d\left(\Delta \sigma^{(q)}\right)(-\boldsymbol{k}),
$$

since it is a real Lorentz-scalar momentum-space quantity. Therefore time reversal is equivalent to the $k_{T}$-parity transformation discussed above, and, hence, to the $z \leftrightarrow y$ interchange. Because of the odderon exchange, the interaction with the target $\mathcal{I}_{\text {anti }}^{(q)}(\boldsymbol{x}, \boldsymbol{y}, \boldsymbol{z})$ is $\boldsymbol{z} \leftrightarrow \boldsymbol{y}$ anti-symmetric, and is, therefore, $T$-odd. This results in the spin-dependent cross section being $T$-odd too,

$$
d\left(\Delta \sigma^{(q)}\right)(\boldsymbol{k}) \stackrel{T}{\rightarrow} d\left(\Delta \sigma^{(q)}\right)(-\boldsymbol{k})=-d\left(\Delta \sigma^{(q)}\right)(\boldsymbol{k}),
$$

leading to the $T$-odd STSA $A_{N}$, in agreement with the standard expectations [27].

It is interesting to note that in the high-energy approximation considered here the application of time reversal to dipole correlators is equivalent to the application of $C$-parity, such that the STSA arises from the odderon exchange, which is both $T$ - and $C$-odd. As one can check explicitly, under time reversal Wilson lines transform as

$$
V_{\boldsymbol{x}} \stackrel{T}{\rightarrow} T V_{\boldsymbol{x}} T^{-1}=\left(V_{\boldsymbol{x}}^{\dagger}\right)^{*}=V_{\boldsymbol{x}}^{T}
$$

with $T$ in the superscript denoting transposition. Note that if the original Wilson line was a future-pointing integral along the $x^{+}$-axis, the time-reversed Wilson line on the right of Eq. (36) can be thought of as a complex conjugate of a past-pointing Wilson line or a transpose of the future-pointing Wilson line. The integration in either interpretation runs along the $x^{-}$axis: we will not show or refer to such $x^{+} \leftrightarrow x^{-}$interchange explicitly, as it can be eliminated by a simple relabeling of the $x^{3}$-axis. Since the averaging over the nuclear target is $T$-even, which can be inferred from [35, 36], the (coordinate-space) time reversal of the odderon amplitude $O_{x} \boldsymbol{y}=\left\langle\hat{O}_{\boldsymbol{x}} \boldsymbol{y}\right\rangle$ applies only to the odderon operator, such that

$$
\left\langle\hat{O}_{\boldsymbol{x} \boldsymbol{y}}\right\rangle \stackrel{T}{\rightarrow}-\left\langle\hat{O}_{\boldsymbol{x} \boldsymbol{y}}\right\rangle,
$$

because $\left\langle\hat{D}_{\boldsymbol{x} y}\right\rangle$ is invariant under transposition and $T$ is antilinear $(i \stackrel{T}{\rightarrow}-i)$. Thus we see that the $T$-odd odderon exchange leads to the $T$-odd STSA observable $A_{N}$.

Finally, the reader may wonder whether the cross section in Eq. (32a) is in fact non-zero. While it is very difficult to carry out the integration in Eq. (32a) exactly, we instead will evaluate Eq. (32a) approximately in Sec. III) showing that the cross section and the corresponding STSA $A_{N}$ are in fact non-zero. However, first we would like to derive the analogues of Eqs. (32) for gluon and prompt photon production.

\footnotetext{
${ }^{2}$ In the past, the relation between the odderon and the single and double transverse spin asymmetries was investigated in 82 86 in the pomeron and reggeon formalism.
} 


\section{STSA in Direct Quark Production}

Another channel for quark production is shown in Fig. 11] and contains a "virtual" gluon correction, without the gluon being present in the final state. The gluon and quark in the loop may still interact with the target, as depicted in Fig. 11. It can be shown that similar diagrams with the gluon loop located either completely before or after the interaction with the target do not contribute to the STSA and are, therefore, not considered here. The process illustrated in Fig. 11 only leads to quark production at $\alpha=1$, and may seem to be negligible if, in order to avoid the projectile's fragmentation region, we constrain ourselves to $\alpha<1$ kinematics for the produced quark. However, remembering that for phenomenological applications one would have to convolute our quark production cross section (32) with the quark distribution in the projectile proton, we see that $\alpha=1$ production may still lead to non-negligible quark production at Feynman- $x$ less than one, possibly avoiding mixing with the proton's fragmentation region.

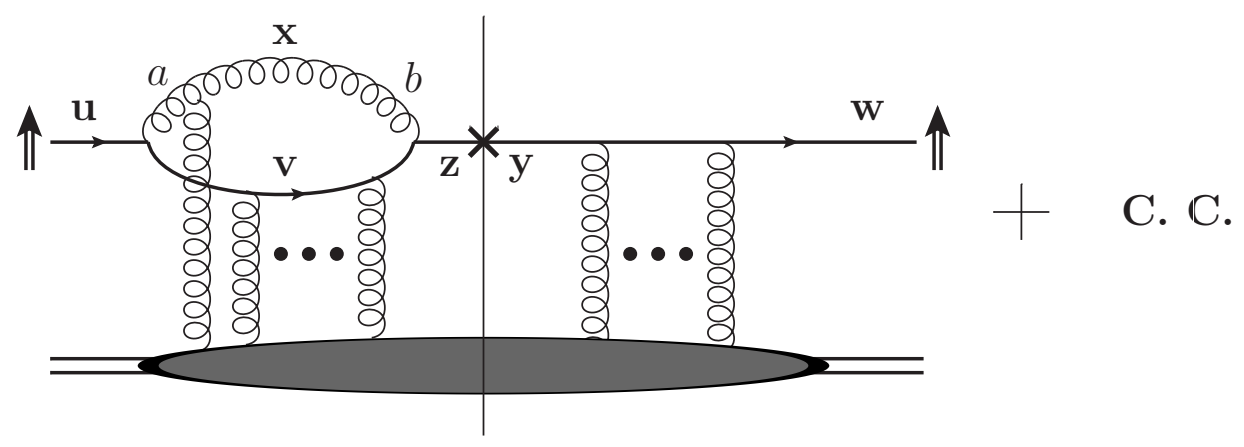

FIG. 11. Direct quark production in the polarized quark-nucleus scattering.

The calculation of the cross section for the process in Fig. 11 can be straightforwardly carried out along the lines specified above, yielding for $\boldsymbol{p}=0$

$$
\begin{aligned}
& \frac{d \sigma^{(q) \text { direct }}}{d^{2} k d y_{q}}=\frac{C_{F}}{(2 \pi)^{2}} \delta(1-\alpha) \int d^{2} x d^{2} y d^{2} z d^{2} u d^{2} v d^{2} w e^{-i \boldsymbol{k} \cdot(\boldsymbol{z}-\boldsymbol{y})} \int_{0}^{1} \frac{d \alpha^{\prime}}{4 \pi\left(1-\alpha^{\prime}\right)} \Phi_{\chi}\left(\boldsymbol{v}-\boldsymbol{x}, \boldsymbol{v}-\boldsymbol{x}, \alpha^{\prime}\right) \\
& \times\left[\delta^{2}\left[(\boldsymbol{u}-\boldsymbol{x})-\alpha^{\prime}(\boldsymbol{v}-\boldsymbol{x})\right] \delta^{2}\left[(\boldsymbol{z}-\boldsymbol{x})-\alpha^{\prime}(\boldsymbol{v}-\boldsymbol{x})\right] \delta^{2}(\boldsymbol{y}-\boldsymbol{w}) \mathcal{I}_{1}^{(q) \operatorname{direct}}(\boldsymbol{x}, \boldsymbol{y}, \boldsymbol{v})\right. \\
& \left.+\delta^{2}\left[(\boldsymbol{w}-\boldsymbol{x})-\alpha^{\prime}(\boldsymbol{v}-\boldsymbol{x})\right] \delta^{2}\left[(\boldsymbol{y}-\boldsymbol{x})-\alpha^{\prime}(\boldsymbol{v}-\boldsymbol{x})\right] \delta^{2}(\boldsymbol{z}-\boldsymbol{u}) \mathcal{I}_{2}^{(q) \operatorname{direct}}(\boldsymbol{x}, \boldsymbol{z}, \boldsymbol{v})\right]
\end{aligned}
$$

with $\alpha^{\prime}$ the longitudinal momentum fraction of the incoming quark carried by the quark in the loop, $y_{q} \sim \ln 1 / \alpha$ (up to an additive term defining the zero rapidity direction), and the interactions with the target

$$
\begin{aligned}
& \mathcal{I}_{1}^{(q) \text { direct }}(\boldsymbol{x}, \boldsymbol{y}, \boldsymbol{v})=\left\langle\frac{N_{c}}{2 C_{F}} \hat{D}_{\boldsymbol{v} \boldsymbol{x}} \hat{D}_{\boldsymbol{x} \boldsymbol{y}}-\frac{1}{2 N_{c} C_{F}} \hat{D}_{\boldsymbol{v} \boldsymbol{y}}\right\rangle, \\
& \mathcal{I}_{2}^{(q){ }^{\text {direct }}(\boldsymbol{x}, \boldsymbol{z}, \boldsymbol{v})}=\left\langle\frac{N_{c}}{2 C_{F}} \hat{D}_{\boldsymbol{x} \boldsymbol{v}} \hat{D}_{\boldsymbol{z} \boldsymbol{x}}-\frac{1}{2 N_{c} C_{F}} \hat{D}_{\boldsymbol{z} \boldsymbol{v}}\right\rangle .
\end{aligned}
$$

To obtain the contribution to the numerator of STSA in Eq. (11) from Eq. (38) we keep the spin-dependent part of the wave function squared, $\Phi_{\text {pol }}$, and anti-symmetrize the integrand with respect to the $\boldsymbol{z} \leftrightarrow \boldsymbol{y}$ interchange. This gives

$$
\begin{aligned}
& d\left(\Delta \sigma^{(q) \text { direct }}\right)=\frac{C_{F}}{2(2 \pi)^{3}} \delta(1-\alpha) \int d^{2} x d^{2} y d^{2} z e^{-i \boldsymbol{k} \cdot(\boldsymbol{z}-\boldsymbol{y})} \int_{0}^{1} \frac{d \alpha^{\prime}}{\left(1-\alpha^{\prime}\right) \alpha^{2}} \\
& \quad \times\left[\Phi_{\text {pol }}\left(\frac{\boldsymbol{z}-\boldsymbol{x}}{\alpha^{\prime}}, \frac{\boldsymbol{z}-\boldsymbol{x}}{\alpha^{\prime}}, \alpha^{\prime}\right) \mathcal{I}_{\text {anti }}^{(q) \text { direct }}(\boldsymbol{x}, \boldsymbol{y}, \boldsymbol{z})-\Phi_{\text {pol }}\left(\frac{\boldsymbol{y}-\boldsymbol{x}}{\alpha^{\prime}}, \frac{\boldsymbol{y}-\boldsymbol{x}}{\alpha^{\prime}}, \alpha^{\prime}\right) \mathcal{I}_{\text {anti }}^{(q) \text { direct }}(\boldsymbol{x}, \boldsymbol{z}, \boldsymbol{y})\right]
\end{aligned}
$$


with the anti-symmetrized interaction given by

$$
\begin{gathered}
\mathcal{I}_{\text {anti }}^{(q) \text { direct }}(\boldsymbol{x}, \boldsymbol{y}, \boldsymbol{z}) \equiv \mathcal{I}_{1}^{(q) \operatorname{direct}}\left(\boldsymbol{x}, \boldsymbol{y}, \boldsymbol{x}+\frac{\boldsymbol{z}-\boldsymbol{x}}{\alpha^{\prime}}\right)-\mathcal{I}_{2}^{(q) \operatorname{direct}}\left(\boldsymbol{x}, \boldsymbol{y}, \boldsymbol{x}+\frac{\boldsymbol{z}-\boldsymbol{x}}{\alpha^{\prime}}\right) \\
=i\left\langle\frac{N_{c}}{C_{F}}\left[\hat{S}_{\boldsymbol{x}, \boldsymbol{y}} \hat{O}_{\boldsymbol{x}+\frac{1}{\alpha^{\prime}}(\boldsymbol{z}-\boldsymbol{x}), \boldsymbol{x}}+\hat{S}_{\boldsymbol{x}, \boldsymbol{x}+\frac{1}{\alpha^{\prime}}(\boldsymbol{z}-\boldsymbol{x})} \hat{O}_{\boldsymbol{x}, \boldsymbol{y}}\right]+\frac{1}{N_{c} C_{F}} \hat{O}_{\boldsymbol{y}, \boldsymbol{x}+\frac{1}{\alpha^{\prime}}(\boldsymbol{z}-\boldsymbol{x})}\right\rangle .
\end{gathered}
$$

Note again that the single transverse spin asymmetry is due to the odderon-mediated interactions with the target.

\section{STSA in Gluon and Photon Production}

Having laid out the methodology in Sections $\amalg B$ and $\coprod$ C, we can now perform similar calculations of STSA for the cases of gluon and photon production.

We begin with the gluon production. The gluon production diagrams are shown in Fig. 12 Since now we tag on the gluon, its transverse-space positions are different on both sides of the cut, now denoted $\boldsymbol{z}$ and $\boldsymbol{y}$, while the untagged quark has the same transverse positions $\boldsymbol{x}$ in the amplitude and in the complex conjugate amplitude. We see that to obtain the gluon production cross section from the quark production expression found in the previous Section, we need to interchange

$$
\boldsymbol{z} \leftrightarrow \boldsymbol{x} \text { and } \boldsymbol{y} \leftrightarrow \boldsymbol{x}
$$

in the wave function and its complex conjugate correspondingly. In addition, since we are interested in the differential cross section per unit gluon rapidity $y_{G}$, we use

$$
d y_{G}=\frac{\alpha}{1-\alpha} d y_{q}
$$

(with $\alpha$ still the fraction of the incoming quark's longitudinal momentum carried by the final state quark).
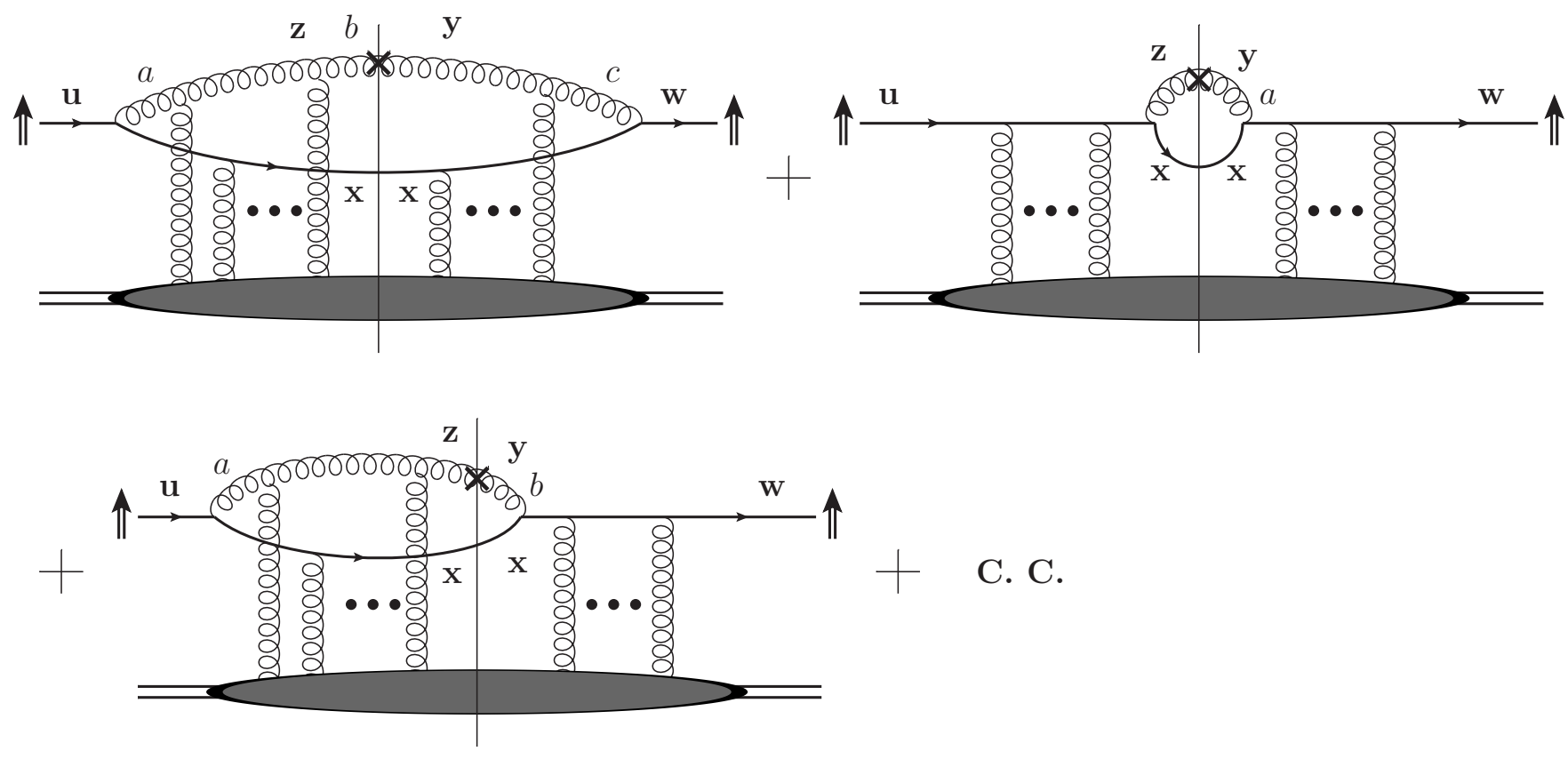

FIG. 12. Diagrams contributing to the gluon / photon production cross section.

These modifications lead to the following expressions for the polarization-dependent and unpolarized cross sections for gluon production

$$
\begin{aligned}
d\left(\Delta \sigma^{(G)}\right) & =\frac{C_{F}}{(2 \pi)^{3}} \int d^{2} x d^{2} y d^{2} z e^{-i \boldsymbol{k} \cdot(\boldsymbol{z}-\boldsymbol{y})} \Phi_{p o l}(\boldsymbol{x}-\boldsymbol{z}, \boldsymbol{x}-\boldsymbol{y}, \alpha) \mathcal{I}_{\text {anti }}^{(G)}(\boldsymbol{x}, \boldsymbol{y}, \boldsymbol{z}) \\
d \sigma_{u n p}^{(G)} & =\frac{C_{F}}{2(2 \pi)^{3}} \int d^{2} x d^{2} y d^{2} z e^{-i \boldsymbol{k} \cdot(\boldsymbol{z}-\boldsymbol{y})} \Phi_{\text {unp }}(\boldsymbol{x}-\boldsymbol{z}, \boldsymbol{x}-\boldsymbol{y}, \alpha) \mathcal{I}_{\text {symm }}^{(G)}(\boldsymbol{x}, \boldsymbol{y}, \boldsymbol{z})
\end{aligned}
$$


with $\Phi_{\text {pol }}$ and $\Phi_{\text {unp }}$ still given by Eqs. (15) and (14).

The interaction with the target for the gluon production case can be calculated along the similar lines to the above calculation of quark production by using Fig. 12, yielding

$$
\mathcal{I}^{(G)}=\left\langle\hat{D}_{\boldsymbol{u} \boldsymbol{w}}+\frac{N_{c}}{2 C_{F}} \hat{D}_{\boldsymbol{z} \boldsymbol{y}} \hat{D}_{\boldsymbol{y} \boldsymbol{z}}-\frac{1}{2 N_{c} C_{F}}-\frac{N_{c}}{2 C_{F}} \hat{D}_{\boldsymbol{x} \boldsymbol{z}} \hat{D}_{\boldsymbol{z} \boldsymbol{w}}+\frac{1}{2 N_{c} C_{F}} \hat{D}_{\boldsymbol{x} \boldsymbol{w}}-\frac{N_{c}}{2 C_{F}} \hat{D}_{\boldsymbol{u} \boldsymbol{y}} \hat{D}_{\boldsymbol{y} \boldsymbol{x}}+\frac{1}{2 N_{c} C_{F}} \hat{D}_{\boldsymbol{u} \boldsymbol{x}}\right\rangle .
$$

Note that now

$$
\begin{aligned}
\boldsymbol{u} & =\boldsymbol{x}+(1-\alpha)(\boldsymbol{z}-\boldsymbol{x}) \\
\boldsymbol{w} & =\boldsymbol{x}+(1-\alpha)(\boldsymbol{y}-\boldsymbol{x})
\end{aligned}
$$

due to the interchanges of Eq. (42) carried out in Eqs. (26).

Separating the interaction into the symmetric and anti-symmetric components under the $\boldsymbol{z} \leftrightarrow \boldsymbol{y}$ interchange one obtains

$$
\begin{aligned}
\mathcal{I}_{s y m m}^{(G)}= & \left\langle\hat{S}_{\boldsymbol{u} \boldsymbol{w}}+\frac{N_{c}}{2 C_{F}}\left(\hat{S}_{\boldsymbol{z} \boldsymbol{y}} \hat{S}_{\boldsymbol{y} \boldsymbol{z}}-\hat{O}_{\boldsymbol{z} \boldsymbol{y}} \hat{O}_{\boldsymbol{y} \boldsymbol{z}}\right)-\frac{1}{2 N_{c} C_{F}}-\frac{N_{c}}{2 C_{F}}\left(\hat{S}_{\boldsymbol{x} \boldsymbol{z}} \hat{S}_{\boldsymbol{z} \boldsymbol{w}}-\hat{O}_{\boldsymbol{x} \boldsymbol{z}} \hat{O}_{\boldsymbol{z} \boldsymbol{w}}\right)+\frac{1}{2 N_{c} C_{F}} \hat{S}_{\boldsymbol{x} \boldsymbol{w}}\right. \\
& \left.-\frac{N_{c}}{2 C_{F}}\left(\hat{S}_{\boldsymbol{u} \boldsymbol{y}} \hat{S}_{\boldsymbol{y} \boldsymbol{x}}-\hat{O}_{\boldsymbol{u} \boldsymbol{y}} \hat{O}_{\boldsymbol{y} \boldsymbol{x}}\right)+\frac{1}{2 N_{c} C_{F}} \hat{S}_{\boldsymbol{u} \boldsymbol{x}}\right\rangle \\
\mathcal{I}_{\text {anti }}^{(G)}= & i\left\langle\hat{O}_{\boldsymbol{u} \boldsymbol{w}}-\frac{N_{c}}{2 C_{F}}\left(\hat{S}_{\boldsymbol{x} \boldsymbol{z}} \hat{O}_{\boldsymbol{z} \boldsymbol{w}}+\hat{O}_{\boldsymbol{x} \boldsymbol{z}} \hat{S}_{\boldsymbol{z} \boldsymbol{w}}\right)+\frac{1}{2 N_{c} C_{F}} \hat{O}_{\boldsymbol{x} \boldsymbol{w}}\right. \\
& \left.-\frac{N_{c}}{2 C_{F}}\left(\hat{S}_{\boldsymbol{u} \boldsymbol{y}} \hat{O}_{\boldsymbol{y} \boldsymbol{x}}+\hat{O}_{\boldsymbol{u} \boldsymbol{y}} \hat{S}_{\boldsymbol{y} \boldsymbol{x}}\right)+\frac{1}{2 N_{c} C_{F}} \hat{O}_{\boldsymbol{u} \boldsymbol{x}}\right\rangle,
\end{aligned}
$$

where we have used the fact that $\hat{O}_{y z}=-\hat{O}_{z y}$ which follows from the definition in Eq. (29c).

Finally, in the large- $N_{c}$ limit Eqs. (47) simplify to

$$
\begin{aligned}
& \left.\mathcal{I}_{\text {symm }}^{(G)}\right|_{\text {large- } N_{c}}=S_{\boldsymbol{u} \boldsymbol{w}}+\left(S_{\boldsymbol{z} \boldsymbol{y}}\right)^{2}-S_{\boldsymbol{x} \boldsymbol{z}} S_{\boldsymbol{z} \boldsymbol{w}}-S_{\boldsymbol{u} \boldsymbol{y}} S_{\boldsymbol{y} \boldsymbol{x}}+\left(O_{\boldsymbol{z} \boldsymbol{y}}\right)^{2}+O_{\boldsymbol{x} \boldsymbol{z}} O_{\boldsymbol{z} \boldsymbol{w}}+O_{\boldsymbol{u} \boldsymbol{y}} O_{\boldsymbol{y} \boldsymbol{x}} \\
& \left.\mathcal{I}_{\text {anti }}^{(G)}\right|_{\text {large- }-N_{c}}=i\left[O_{\boldsymbol{u} \boldsymbol{w}}-S_{\boldsymbol{x} \boldsymbol{z}} O_{\boldsymbol{z} \boldsymbol{w}}-O_{\boldsymbol{x} \boldsymbol{z}} S_{\boldsymbol{z} \boldsymbol{w}}-S_{\boldsymbol{u} \boldsymbol{y}} O_{\boldsymbol{y} \boldsymbol{x}}-O_{\boldsymbol{u} \boldsymbol{y}} S_{\boldsymbol{y} \boldsymbol{x}}\right] .
\end{aligned}
$$

Eqs. (47) and (44), when used in Eq. (11), give an expression for the gluon STSA in the CGC formalism. This is another main result of this work.

Constructing the cross sections for prompt photon production out of the gluon production cross sections we have just derived is straightforward. One has to drop all color factors in the light-cone wave functions, replace $\alpha_{s} \rightarrow \alpha_{E M} Z_{f}^{2}$ with $Z_{f}$ the electric charge of a quark with flavor $f$ in units of the electron charge, and recalculate the interaction with the target remembering that the photon, in this lowest order in $\alpha_{E M}$ approximation does not interact. One obtains the polarization-dependent and unpolarized cross sections for photon production

$$
\begin{aligned}
d\left(\Delta \sigma^{(\gamma)}\right) & =\frac{1}{(2 \pi)^{3}} \int d^{2} x d^{2} y d^{2} z e^{-i \boldsymbol{k} \cdot(\boldsymbol{z}-\boldsymbol{y})} \Phi_{\text {pol }}(\boldsymbol{x}-\boldsymbol{z}, \boldsymbol{x}-\boldsymbol{y}, \alpha) \mathcal{I}_{\text {anti }}^{(\gamma)}(\boldsymbol{x}, \boldsymbol{y}, \boldsymbol{z}) \\
d \sigma_{u n p}^{(\gamma)} & =\frac{1}{2(2 \pi)^{3}} \int d^{2} x d^{2} y d^{2} z e^{-i \boldsymbol{k} \cdot(\boldsymbol{z}-\boldsymbol{y})} \Phi_{\text {unp }}(\boldsymbol{x}-\boldsymbol{z}, \boldsymbol{x}-\boldsymbol{y}, \alpha) \mathcal{I}_{\text {symm }}^{(\gamma)}(\boldsymbol{x}, \boldsymbol{y}, \boldsymbol{z}),
\end{aligned}
$$

where $\Phi_{\text {pol }}$ and $\Phi_{\text {unp }}$ are given by Eqs. (15) and (14) with the $\alpha_{s} \rightarrow \alpha_{E M} Z_{f}^{2}$ replacement.

The interaction with the target is calculated to be

$$
\mathcal{I}^{(\gamma)}=1+D_{\boldsymbol{u} \boldsymbol{w}}-D_{\boldsymbol{x} \boldsymbol{w}}-D_{\boldsymbol{u} \boldsymbol{x}}
$$

with the symmetric and anti-symmetric under $\boldsymbol{z} \leftrightarrow \boldsymbol{y}$ parts

$$
\begin{aligned}
\mathcal{I}_{\text {symm }}^{(\gamma)} & =1+S_{\boldsymbol{u} \boldsymbol{w}}-S_{\boldsymbol{x} \boldsymbol{w}}-S_{\boldsymbol{u} \boldsymbol{x}} \\
\mathcal{I}_{\text {anti }}^{(\gamma)} & =i\left[O_{\boldsymbol{u} \boldsymbol{w}}-O_{\boldsymbol{x} \boldsymbol{w}}-O_{\boldsymbol{u} \boldsymbol{x}}\right] .
\end{aligned}
$$

Eqs. (51) and (49) along with Eq. (11) give us the prompt photon STSA. This is the third and final main formal result of this work. Note that below we will show that Eq. (49a) leads to $d\left(\Delta \sigma^{(\gamma)}\right)=0$ for any target, which implies zero STSA for photons in our mechanism. 
We have constructed general expressions for STSA generated by quark, gluon, and photon production in $q^{\uparrow}+A$ collisions. Knowing the light-cone wave functions squared (14), (15) and the interactions for the 3 channels (30) (along with (41)), (47), (51) one can make explicit predictions for the corresponding asymmetries. In general terms, we have shown that in this formalism the asymmetry is generated by coupling of the spin-dependent part of the wave function to the Odderon interaction with the target.

\section{EVALUATIONS AND ESTIMATES OF THE ASYMMETRY}

Unfortunately, Eqs. (32), (44), and (49) are too complicated to be integrated out analytically in the general case. In this Section, in order to understand the qualitative behavior of our results, we evaluate the integrals analytically, taking the interaction with the target in the quasi-classical Glauber-Mueller approximation. In such a quasi-classical limit, the real part of the $S$ matrix (29b) is 75]

$$
S_{\boldsymbol{x} \boldsymbol{y}}=\exp \left[-\frac{1}{4}|\boldsymbol{x}-\boldsymbol{y}|^{2} Q_{s}^{2}\left(\frac{\boldsymbol{x}+\boldsymbol{y}}{2}\right) \ln \frac{1}{|\boldsymbol{x}-\boldsymbol{y}| \Lambda}\right],
$$

where the quark saturation scale scale $Q^{2}(\boldsymbol{b})$ is defined in terms of the nuclear profile function (transverse nuclear density) $T(\boldsymbol{b})$ as

$$
Q_{s}^{2}(\boldsymbol{b}) \equiv \frac{4 \pi \alpha_{s}^{2} C_{F}}{N_{c}} T(\boldsymbol{b})
$$

and $\Lambda$ is a non-perturbative IR cutoff.

In the same quasi-classical approximation the odderon amplitude is 71

$$
O_{\boldsymbol{x} \boldsymbol{y}}=\left\langle c_{0} \alpha_{s}^{3} \ln ^{3} \frac{|\boldsymbol{x}-\boldsymbol{r}|}{|\boldsymbol{y}-\boldsymbol{r}|}\right\rangle \exp \left[-\frac{1}{4}|\boldsymbol{x}-\boldsymbol{y}|^{2} Q_{s}^{2}\left(\frac{\boldsymbol{x}+\boldsymbol{y}}{2}\right) \ln \frac{1}{|\boldsymbol{x}-\boldsymbol{y}| \Lambda}\right]
$$

with the constant 72,74$]^{3}$

$$
c_{0}=-\frac{\left(N_{c}^{2}-4\right)\left(N_{c}^{2}-1\right)}{12 N_{c}^{3}} .
$$

The logarithm cubed in Eq. (54) arises due to the triple gluon exchange between the dipole and some quark in the target nucleus located at transverse position $\boldsymbol{r}$. Angle brackets in Eq. (54) denote the averaging over positions of the quark in the nuclear wave function, along with the summation over all the nucleons in the nucleus that may contain this quark. This averaging is carried out in Appendix A, yielding

$$
O_{x \boldsymbol{y}} \approx-c_{0} \alpha_{s}^{3} \frac{3 \pi}{16}|\boldsymbol{x}-\boldsymbol{y}|^{2} \exp \left[-\frac{1}{4}|\boldsymbol{x}-\boldsymbol{y}|^{2} Q_{s}^{2}\left(\frac{\boldsymbol{x}+\boldsymbol{y}}{2}\right) \ln \frac{1}{|\boldsymbol{x}-\boldsymbol{y}| \Lambda}\right](\boldsymbol{x}-\boldsymbol{y}) \cdot \nabla T\left(\frac{\boldsymbol{x}+\boldsymbol{y}}{2}\right) .
$$

For simplicity we will also work in the large- $N_{c}$ limit for the light-cone wave function. Just like before, we mainly concentrate on the quark production case in Eq. (32): STSA in the gluon production channel can be evaluated along similar lines. We will also consider STSA for the prompt photon production.

\section{A. Single Transverse Spin Asymmetry in Quark Production}

\section{Spin-Dependent Quark Production Cross Section}

First let us evaluate the numerator of the STSA in Eq. (11), which, in the quark production case, is given by Eq. (32a). (For simplicity we assume that $\alpha<1$ which allows us to drop the contribution from Eq. (40).) Working

\footnotetext{
${ }^{3}$ Note that the sign is different from that in [72, 74]: the sign in Eq. (55) arises when using a consistent convention for the sign of the coupling $g$ both in the Wilson lines and in the classical gluon field of the target. (Our sign convention is to have $+i g$ for the quark-gluon vertex, resulting in $+i g$ in the Wilson lines (16).) While the physical conclusions reached in 71 74 $]$ are independent of the sign of the odderon amplitude, the direction of the asymmetry in question explicitly depends on the sign of $O_{\boldsymbol{x}} \boldsymbol{y}$.
} 
in the large- $N_{c}$ limit for the light-cone wave function we substitute the interaction from Eq. (31b) into Eq. (32a) to obtain

$$
\begin{gathered}
d\left(\Delta \sigma^{(q)}\right)=i \frac{N_{c}}{2(2 \pi)^{3}} \frac{\alpha}{1-\alpha} \int d^{2} x d^{2} y d^{2} z e^{-i \boldsymbol{k} \cdot(\boldsymbol{z}-\boldsymbol{y})} \Phi_{p o l}(\boldsymbol{z}-\boldsymbol{x}, \boldsymbol{y}-\boldsymbol{x}, \alpha)\left[O_{\boldsymbol{z} \boldsymbol{y}}+O_{\boldsymbol{u} \boldsymbol{w}}-O_{\boldsymbol{z} \boldsymbol{x}} S_{\boldsymbol{x} \boldsymbol{w}}-O_{\boldsymbol{u} \boldsymbol{x}} S_{\boldsymbol{x} \boldsymbol{y}}\right. \\
\left.-S_{\boldsymbol{z} \boldsymbol{x}} O_{\boldsymbol{x} \boldsymbol{w}}-S_{\boldsymbol{u} \boldsymbol{x}} O_{\boldsymbol{x} \boldsymbol{y}}\right] .
\end{gathered}
$$

Our goal now is to evaluate this expression using the $S$-matrix from Eq. (52) and the odderon amplitude (56).

The interaction with the target in Eq. (57) is non-linear. It is tempting to try to simplify the problem by neglecting all the multiple rescattering saturation effects. In such a linearized approximation Eq. (57) reduces to

$$
\begin{array}{r}
d\left(\Delta \sigma^{(q)}\right)_{l i n}=i \frac{N_{c}}{2(2 \pi)^{3}} \frac{\alpha}{1-\alpha} \int d^{2} x d^{2} y d^{2} z e^{-i \boldsymbol{k} \cdot(\boldsymbol{z}-\boldsymbol{y})} \Phi_{p o l}(\boldsymbol{z}-\boldsymbol{x}, \boldsymbol{y}-\boldsymbol{x}, \alpha) \\
\times\left[o_{\boldsymbol{z} \boldsymbol{y}}+o_{\boldsymbol{u} \boldsymbol{w}}-o_{\boldsymbol{z} \boldsymbol{x}}-o_{\boldsymbol{u} \boldsymbol{x}}-o_{\boldsymbol{x} \boldsymbol{w}}-o_{\boldsymbol{x} \boldsymbol{y}}\right]
\end{array}
$$

where

$$
o_{\boldsymbol{x} \boldsymbol{y}} \approx \alpha_{s}^{3} \frac{\pi N_{c}}{64}|\boldsymbol{x}-\boldsymbol{y}|^{2}(\boldsymbol{x}-\boldsymbol{y}) \cdot \boldsymbol{\nabla} T\left(\frac{\boldsymbol{x}+\boldsymbol{y}}{2}\right)
$$

is the linear part of the averaged odderon amplitude (56). However, one can easily show that the cross section in Eq. (58) is in fact zero, i.e., that

$$
d\left(\Delta \sigma^{(q)}\right)_{l i n}=0 .
$$

We illustrate this by considering the $o_{\boldsymbol{z} y}$ term in Eq. (58). Defining new transverse vectors

$$
\tilde{z}=\boldsymbol{z}-\boldsymbol{x}, \quad \tilde{\boldsymbol{y}}=\boldsymbol{y}-\boldsymbol{x},
$$

we rewrite the $o_{\boldsymbol{z} y}$ contribution to the cross section in Eq. (58) as

$$
i \frac{N_{c}}{2(2 \pi)^{3}} \frac{\alpha}{1-\alpha} \int d^{2} \tilde{y} d^{2} \tilde{z} e^{-i \boldsymbol{k} \cdot(\tilde{\boldsymbol{z}}-\tilde{\boldsymbol{y}})} \Phi_{p o l}(\tilde{\boldsymbol{z}}, \tilde{\boldsymbol{y}}, \alpha) \int d^{2} x o_{\tilde{\boldsymbol{z}}+\boldsymbol{x}, \tilde{\boldsymbol{y}}+\boldsymbol{x}}
$$

This expression is zero since

$$
\int d^{2} x o_{\tilde{\boldsymbol{z}}+\boldsymbol{x}, \tilde{\boldsymbol{y}}+\boldsymbol{x}}=0
$$

due to the fact that the odderon amplitude (29c) (and, therefore, the linearized odderon amplitude (59)) is an antisymmetric function of its transverse coordinate arguments,

$$
O_{x y}=-O_{y x}
$$

The argument goes as follows. Employing Eq. (64) and shifting the integration variables we write

$$
f(\boldsymbol{y}) \equiv \int d^{2} x O_{\boldsymbol{x}, \boldsymbol{x}+\boldsymbol{y}}=-\int d^{2} x O_{\boldsymbol{x}, \boldsymbol{x}-\boldsymbol{y}}=-f(-\boldsymbol{y}) .
$$

Since $f(\boldsymbol{y})$ depends only on one vector $\boldsymbol{y}$ and is a scalar under the rotations in the transverse plane, it is a function of $\boldsymbol{y}^{2}$ only, and can satisfy (65) (i.e., can be an odd function of $\boldsymbol{y}$ ) only if $f(\boldsymbol{y})=0$. This demonstrates that

$$
\int d^{2} x O_{\boldsymbol{x}, \boldsymbol{x}+\boldsymbol{y}}=0 .
$$

Similar arguments can be carried out for other terms in Eq. (58), leading in the end to Eq. (60). We arrive at an important conclusion: STSA cannot result from the interaction with the target mediated by the odderon exchange alone. Neglecting the interactions contained in the dipole $S$-matrices in Eq. (57) would lead to zero transverse spin asymmetry. This is an important observation elucidating the nature of our result (32) and the corresponding STSA: in order to generate a non-zero STSA the interaction with the target has to contain both the $C$-odd and $C$-even contributions! 
Returning to the general case of Eq. (57) we see that the argument we have just presented demonstrates that the $O_{z y}$ and $O_{\boldsymbol{u} w}$ terms are zero in the general case as well, since they are not multiplied by the $S$-matrices. Dropping these terms yields

$$
\begin{array}{r}
d\left(\Delta \sigma^{(q)}\right)=-i \frac{N_{c}}{2(2 \pi)^{3}} \frac{\alpha}{1-\alpha} \int d^{2} x d^{2} y d^{2} z e^{-i \boldsymbol{k} \cdot(\boldsymbol{z}-\boldsymbol{y})} \Phi_{p o l}(\boldsymbol{z}-\boldsymbol{x}, \boldsymbol{y}-\boldsymbol{x}, \alpha) \\
\times\left[O_{\boldsymbol{z} \boldsymbol{x}} S_{\boldsymbol{x} \boldsymbol{w}}+O_{\boldsymbol{u} \boldsymbol{x}} S_{\boldsymbol{x} \boldsymbol{y}}+O_{\boldsymbol{x} \boldsymbol{w}} S_{\boldsymbol{z} \boldsymbol{x}}+O_{\boldsymbol{x} \boldsymbol{y}} S_{\boldsymbol{u} \boldsymbol{x}}\right] .
\end{array}
$$

To evaluate Eq. (67) let us first study its large- $k_{T}$ asymptotics. Since $\tilde{m} \leq m$ and the quark mass $m$ is at most the constituent quark mass of about $300 \mathrm{MeV}$ (we assume light quark flavors), we have $k_{T} \gg Q_{s} \gg \tilde{m}$. Changing the coordinates using Eq. (61) reduces it to

$$
\begin{aligned}
d\left(\Delta \sigma^{(q)}\right) & =-i \frac{N_{c}}{2(2 \pi)^{3}} \frac{\alpha}{1-\alpha} \int d^{2} x d^{2} \tilde{y} d^{2} \tilde{z} e^{-i \boldsymbol{k} \cdot(\tilde{\boldsymbol{z}}-\tilde{\boldsymbol{y}})} \Phi_{p o l}(\tilde{\boldsymbol{z}}, \tilde{\boldsymbol{y}}, \alpha) \\
& \times\left[O_{\boldsymbol{x}+\tilde{\boldsymbol{z}}, \boldsymbol{x}} S_{\boldsymbol{x}, \boldsymbol{x}+\alpha \tilde{\boldsymbol{y}}}+O_{\boldsymbol{x}+\alpha \tilde{\boldsymbol{z}}, \boldsymbol{x}} S_{\boldsymbol{x}, \boldsymbol{x}+\tilde{\boldsymbol{y}}}+O_{\boldsymbol{x}, \boldsymbol{x}+\alpha \tilde{\boldsymbol{y}}} S_{\boldsymbol{x}, \boldsymbol{x}+\tilde{\boldsymbol{z}}}+O_{\boldsymbol{x}, \boldsymbol{x}+\tilde{\boldsymbol{y}}} S_{\boldsymbol{x}, \boldsymbol{x}+\alpha \tilde{\boldsymbol{z}}}\right] .
\end{aligned}
$$

For each term in the square brackets of Eq. (68) the integrals over $\tilde{\boldsymbol{z}}$ and $\tilde{\boldsymbol{y}}$ factorize: taking the large- $k_{T}$ limit in each of them separately, we see that the large- $k_{T}$ asymptotics corresponds to small $\tilde{z}_{T}$ and $\tilde{y}_{T}$. We thus need to expand the interaction with the target term in the square brackets of Eq. (68) to the lowest non-trivial order in $\tilde{z}_{T}$ and $\tilde{y}_{T}$. Note that above we have seen that if we keep the dipole $S$-matrices at the lowest order in the dipole size, $S=1$, then the spin-dependent cross section would be zero. We thus use Eqs. (52) and (56) to expand the $S$-matrices to the next-to-lowest order, while keeping the odderon amplitudes at the lowest order given by Eq. (59). Performing the expansion, substituting the wave function squared from Eq. (15) (also expanded to the lowest non-trivial order in $\tilde{z}_{T}$ and $\tilde{y}_{T}$ ) into Eq. (68), and employing Eq. (53) we obtain

$$
\begin{aligned}
& \left.d\left(\Delta \sigma^{(q)}\right)\right|_{k_{T} \gg Q_{s}} \approx i \frac{N_{c}^{2}}{1024 \pi^{2}} \alpha_{s}^{6} \tilde{m} \alpha^{4} \int d^{2} x d^{2} \tilde{y} d^{2} \tilde{z} e^{-i \boldsymbol{k} \cdot(\tilde{\boldsymbol{z}}-\tilde{\boldsymbol{y}})}\left(\frac{\tilde{z}^{2}}{\tilde{z}_{T}^{2}} \ln \frac{1}{\tilde{m} \tilde{y}_{T}}+\frac{\tilde{y}^{2}}{\tilde{y}_{T}^{2}} \ln \frac{1}{\tilde{m} \tilde{z}_{T}}\right) \tilde{z}_{T}^{2} \tilde{y}_{T}^{2} \\
& \times\left[\tilde{\boldsymbol{z}} \cdot \boldsymbol{\nabla} T\left(\boldsymbol{x}+\frac{\tilde{\boldsymbol{z}}}{2}\right) T\left(\boldsymbol{x}+\frac{\alpha \tilde{\boldsymbol{y}}}{2}\right) \ln \frac{1}{\alpha \tilde{y}_{T} \Lambda}+\alpha \tilde{\boldsymbol{z}} \cdot \boldsymbol{\nabla} T\left(\boldsymbol{x}+\frac{\alpha \tilde{\boldsymbol{z}}}{2}\right) T\left(\boldsymbol{x}+\frac{\tilde{\boldsymbol{y}}}{2}\right) \ln \frac{1}{\tilde{y}_{T} \Lambda}-(\tilde{\boldsymbol{z}} \leftrightarrow \tilde{\boldsymbol{y}})\right] .
\end{aligned}
$$

Since $\tilde{z}_{T}$ and $\tilde{y}_{T}$ are small, one may think of neglecting them compared to $\boldsymbol{x}$ in the arguments of $T$ 's in Eq. (69). However, this would lead to a zero answer after integration over $\boldsymbol{x}$. The reason for this conclusion is that any unpolarized target, after averaging over many events, is rotationally symmetric in the transverse plane. This implies that $\boldsymbol{\nabla} T(\boldsymbol{x})=\boldsymbol{\nabla} T\left(x_{T}\right)=\hat{x} T^{\prime}\left(x_{T}\right)$ where $\hat{x}$ is a unit vector in the direction of $\boldsymbol{x}$ and $T^{\prime}\left(x_{T}\right)=d T\left(x_{T}\right) / d x_{T}$. Integrating $\hat{x}$ over the angles of $\boldsymbol{x}$ would give zero.

Instead of neglecting $\tilde{z}_{T}$ and $\tilde{y}_{T}$, we shift $\boldsymbol{x} \rightarrow \boldsymbol{x}-\tilde{\boldsymbol{z}} / 2$ in the first term in the square brackets of Eq. (69) and expand $T$ along the lines of Eq. (A7), and perform similar operations to the other terms in the brackets obtaining

$$
\begin{aligned}
& \left.d\left(\Delta \sigma^{(q)}\right)\right|_{k_{T} \gg Q_{s}} \approx i \frac{N_{c}^{2}}{2048 \pi^{2}} \alpha_{s}^{6} \tilde{m} \alpha^{4} \int d^{2} x d^{2} y d^{2} z e^{-i \boldsymbol{k} \cdot(\boldsymbol{z}-\boldsymbol{y})}\left(\frac{z^{2}}{z_{T}^{2}} \ln \frac{1}{\tilde{m} y_{T}}+\frac{y^{2}}{y_{T}^{2}} \ln \frac{1}{\tilde{m} z_{T}}\right) z_{T}^{2} y_{T}^{2} \\
& \times\left[\boldsymbol{z} \cdot \boldsymbol{\nabla} T(\boldsymbol{x})(\alpha \boldsymbol{y}-\boldsymbol{z}) \cdot \boldsymbol{\nabla} T(\boldsymbol{x}) \ln \frac{1}{\alpha y_{T} \Lambda}+\alpha \boldsymbol{z} \cdot \boldsymbol{\nabla} T(\boldsymbol{x})(\boldsymbol{y}-\alpha \boldsymbol{z}) \cdot \boldsymbol{\nabla} T(\boldsymbol{x}) \ln \frac{1}{y_{T} \Lambda}-(\boldsymbol{z} \leftrightarrow \boldsymbol{y})\right],
\end{aligned}
$$

where we have dropped the tildes over $\boldsymbol{y}$ and $\boldsymbol{z}$, since now it would not cause confusion.

Using $\boldsymbol{\nabla} T(\boldsymbol{x})=\boldsymbol{\nabla} T\left(x_{T}\right)=\hat{x} T^{\prime}\left(x_{T}\right)$ and integrating over the angles of $\boldsymbol{x}$ reduces Eq. (70) to

$$
\begin{aligned}
& \left.d\left(\Delta \sigma^{(q)}\right)\right|_{k_{T} \gg Q_{s}} \approx i \frac{N_{c}^{2}}{4096 \pi} \alpha_{s}^{6} \tilde{m} \alpha^{4} \int_{0}^{\infty} d x_{T}^{2}\left[T^{\prime}\left(x_{T}\right)\right]^{2} \int d^{2} y d^{2} z e^{-i \boldsymbol{k} \cdot(\boldsymbol{z}-\boldsymbol{y})}\left(\frac{z^{2}}{z_{T}^{2}} \ln \frac{1}{\tilde{m} y_{T}}+\frac{y^{2}}{y_{T}^{2}} \ln \frac{1}{\tilde{m} z_{T}}\right) z_{T}^{2} y_{T}^{2} \\
& \times\left[\boldsymbol{z} \cdot(\alpha \boldsymbol{y}-\boldsymbol{z}) \ln \frac{1}{\alpha y_{T} \Lambda}+\alpha \boldsymbol{z} \cdot(\boldsymbol{y}-\alpha \boldsymbol{z}) \ln \frac{1}{y_{T} \Lambda}-\alpha \boldsymbol{y} \cdot(\boldsymbol{z}-\alpha \boldsymbol{y}) \ln \frac{1}{z_{T} \Lambda}-\boldsymbol{y} \cdot(\alpha \boldsymbol{z}-\boldsymbol{y}) \ln \frac{1}{\alpha z_{T} \Lambda}\right] .
\end{aligned}
$$

Integrating over $\boldsymbol{y}$ and $\boldsymbol{z}$ in Eq. (71) and discarding delta-functions of $\boldsymbol{k}$ (since $k_{T} \neq 0$ ) yields

$$
\left.d\left(\Delta \sigma^{(q)}\right)\right|_{k_{T} \gg Q_{s}} \approx \frac{\pi N_{c}^{2}}{8} \alpha_{s}^{6} \tilde{m} \alpha^{4}\left(2+3 \alpha+2 \alpha^{2}\right) \int_{0}^{\infty} d x_{T}^{2}\left[T^{\prime}\left(x_{T}\right)\right]^{2} \frac{k^{2}}{k_{T}^{10}} .
$$

We see that the polarized spectrum falls off rather steeply with $k_{T}$, scaling as $1 / k_{T}^{9}$. This indicates that in the standard collinear factorization framework our STSA generating mechanism originates in some higher-twist operator. 
Another important qualitative feature one can see in Eq. (72) is that the spin-dependent cross section falls off with decreasing longitudinal momentum fraction $\alpha$, which implies that the corresponding STSA decreases with decreasing Feynman- $x$ of the projectile, in qualitative agreement with the experimental data.

To improve on Eq. (72) let us find the spin-dependent differential cross section $d\left(\Delta \sigma^{(q)}\right)$ for lower $k_{T}$, closer to the saturation scale. To be more specific let us relax the $k_{T} \gg Q_{s}$ restriction and consider a broader region of $k_{T} \lesssim Q_{s}$ and $k_{T} \gtrsim Q_{s}$, but still with $k_{T} \gg \tilde{m}$. For such not very large $k_{T}$ we can neglect the logarithms in the exponents of Eqs. (52) and (56) as slowly varying functions compared to powers they multiply [56, 76, 87], writing

$$
S_{\boldsymbol{x} \boldsymbol{y}} \approx \exp \left[-\frac{1}{4}|\boldsymbol{x}-\boldsymbol{y}|^{2} Q_{s}^{2}\left(\frac{\boldsymbol{x}+\boldsymbol{y}}{2}\right)\right]
$$

and

$$
O_{\boldsymbol{x} \boldsymbol{y}} \approx-c_{0} \alpha_{s}^{3} \frac{3 \pi}{16}|\boldsymbol{x}-\boldsymbol{y}|^{2} \exp \left[-\frac{1}{4}|\boldsymbol{x}-\boldsymbol{y}|^{2} Q_{s}^{2}\left(\frac{\boldsymbol{x}+\boldsymbol{y}}{2}\right)\right](\boldsymbol{x}-\boldsymbol{y}) \cdot \boldsymbol{\nabla} T\left(\frac{\boldsymbol{x}+\boldsymbol{y}}{2}\right) .
$$

Substituting Eqs. (73) and (74) into Eq. (68), expanding the polarized wave function squared, and dropping the tildes yields

$$
\begin{aligned}
& d\left(\Delta \sigma^{(q)}\right) \approx-i \frac{N_{c}^{2}}{512 \pi^{3}} \alpha_{s}^{4} \tilde{m} \alpha^{2} \int d^{2} x d^{2} y d^{2} z e^{-i \boldsymbol{k} \cdot(\boldsymbol{z}-\boldsymbol{y})}\left(\frac{z^{2}}{z_{T}^{2}} \ln \frac{1}{\tilde{m} y_{T}}+\frac{y^{2}}{y_{T}^{2}} \ln \frac{1}{\tilde{m} z_{T}}\right)\left[z_{T}^{2} \boldsymbol{z} \cdot \boldsymbol{\nabla} T\left(\boldsymbol{x}+\frac{\boldsymbol{z}}{2}\right)\right. \\
\times & \left.e^{-\frac{1}{4} z_{T}^{2} Q_{s}^{2}\left(\boldsymbol{x}+\frac{z}{2}\right)-\frac{1}{4} \alpha^{2} y_{T}^{2} Q_{s}^{2}\left(\boldsymbol{x}+\frac{\alpha \boldsymbol{y}}{2}\right)}+\alpha^{3} z_{T}^{2} \boldsymbol{z} \cdot \boldsymbol{\nabla} T\left(\boldsymbol{x}+\frac{\alpha \boldsymbol{z}}{2}\right) e^{-\frac{1}{4} \alpha^{2} z_{T}^{2} Q_{s}^{2}\left(\boldsymbol{x}+\frac{\alpha \boldsymbol{z}}{2}\right)-\frac{1}{4} y_{T}^{2} Q_{s}^{2}\left(\boldsymbol{x}+\frac{y}{2}\right)}-(\boldsymbol{z} \leftrightarrow \boldsymbol{y})\right] .
\end{aligned}
$$

Similar to the large- $k_{T}$ asymptotics, we shift $\boldsymbol{x} \rightarrow \boldsymbol{x}-\boldsymbol{z} / 2$ in the first term in the square brackets of Eq. (75) and expand the resulting exponential with the help of Eq. (53) as

$$
e^{-\frac{1}{4} z_{T}^{2} Q_{s}^{2}(\boldsymbol{x})-\frac{1}{4} \alpha^{2} y_{T}^{2} Q_{s}^{2}\left(\boldsymbol{x}+\frac{\alpha y-z}{2}\right)} \approx\left[1-\frac{\pi}{4} \alpha_{s}^{2} \alpha^{2} y_{T}^{2}(\alpha \boldsymbol{y}-\boldsymbol{z}) \cdot \nabla T(\boldsymbol{x})\right] e^{-\frac{1}{4} z_{T}^{2} Q_{s}^{2}(\boldsymbol{x})-\frac{1}{4} \alpha^{2} y_{T}^{2} Q_{s}^{2}(\boldsymbol{x})} .
$$

The 1 in the square brackets of Eq. (76) does not contribute as its contribution vanishes after integration over the angles of $\boldsymbol{x}$ in Eq. (75), leaving only the second term to contribute. Performing similar expansions in the other terms in the square brackets of Eq. (75) we obtain

$$
\begin{aligned}
& d\left(\Delta \sigma^{(q)}\right) \approx i \frac{N_{c}^{2}}{2048 \pi^{2}} \alpha_{s}^{6} \tilde{m} \alpha^{4} \int d^{2} x d^{2} y d^{2} z e^{-i \boldsymbol{k} \cdot(\boldsymbol{z}-\boldsymbol{y})}\left(\frac{z^{2}}{z_{T}^{2}}+\frac{y^{2}}{y_{T}^{2}}\right) z_{T}^{2} y_{T}^{2}[\boldsymbol{z} \cdot \boldsymbol{\nabla} T(\boldsymbol{x})(\alpha \boldsymbol{y}-\boldsymbol{z}) \cdot \nabla T(\boldsymbol{x}) \\
\times & \left.e^{-\frac{1}{4} z_{T}^{2} Q_{s}^{2}(\boldsymbol{x})-\frac{1}{4} \alpha^{2} y_{T}^{2} Q_{s}^{2}(\boldsymbol{x})}+\alpha \boldsymbol{z} \cdot \boldsymbol{\nabla} T(\boldsymbol{x})(\boldsymbol{y}-\alpha \boldsymbol{z}) \cdot \boldsymbol{\nabla} T(\boldsymbol{x}) e^{-\frac{1}{4} \alpha^{2} z_{T}^{2} Q_{s}^{2}(\boldsymbol{x})-\frac{1}{4} y_{T}^{2} Q_{s}^{2}(\boldsymbol{x})}-(\boldsymbol{z} \leftrightarrow \boldsymbol{y})\right],
\end{aligned}
$$

where we have also dropped $\ln 1 / \tilde{m} y_{T}$ and $\ln 1 / \tilde{m} z_{T}$, since, with our precision, similar logarithms were neglected in Eqs. (73) and (74) above as slowly varying functions of their arguments 4 . Again, integrating over the angles of $\boldsymbol{x}$ yields

$$
\begin{aligned}
& d\left(\Delta \sigma^{(q)}\right) \approx i \frac{N_{c}^{2}}{4096 \pi} \alpha_{s}^{6} \tilde{m} \alpha^{4} \int_{0}^{\infty} d x_{T}^{2}\left[T^{\prime}\left(x_{T}\right)\right]^{2} \int d^{2} y d^{2} z e^{-i \boldsymbol{k} \cdot(\boldsymbol{z}-\boldsymbol{y})}\left(\frac{z^{2}}{z_{T}^{2}}+\frac{y^{2}}{y_{T}^{2}}\right) z_{T}^{2} y_{T}^{2} \\
\times & {\left[\left(\alpha^{2} y_{T}^{2}-z_{T}^{2}\right) e^{-\frac{1}{4} z_{T}^{2} Q_{s}^{2}\left(x_{T}\right)-\frac{1}{4} \alpha^{2} y_{T}^{2} Q_{s}^{2}\left(x_{T}\right)}+\left(y_{T}^{2}-\alpha^{2} z_{T}^{2}\right) e^{-\frac{1}{4} \alpha^{2} z_{T}^{2} Q_{s}^{2}\left(x_{T}\right)-\frac{1}{4} y_{T}^{2} Q_{s}^{2}\left(x_{T}\right)}\right] . }
\end{aligned}
$$

Integrating over $\boldsymbol{y}$ and $\boldsymbol{z}$ we get

$$
d\left(\Delta \sigma^{(q)}\right) \approx \frac{\pi N_{c}^{2}}{4} \frac{\alpha_{s}^{6} \tilde{m}}{\alpha^{4}} \int_{0}^{\infty} d x_{T}^{2}\left[T^{\prime}\left(x_{T}\right)\right]^{2} \frac{k^{2} k_{T}^{2}}{Q_{s}^{14}\left(x_{T}\right)}\left[\left(1-\alpha^{2}\right)^{2} k_{T}^{2}-\alpha^{2}\left(1+\alpha^{2}\right) Q_{s}^{2}\left(x_{T}\right)\right] e^{-\frac{k_{T}^{2}}{Q_{s}^{2}\left(x_{T}\right)}\left(1+\frac{1}{\alpha^{2}}\right)} .
$$

This is the final expression for the STSA-generating cross section for quark production. Note again that $k^{2}$ is the $y$-component of the quark's transverse momentum $\boldsymbol{k}=\left(k^{1}, k^{2}\right)$. Let us point out a few of the important features of Eq. (79). First of all, we see that similar to Eq. (72) decreases with decreasing $\alpha$ for small $\alpha$, now due to the factor of $1 / \alpha^{2}$ in the exponent. We also see that for $k_{T} \rightarrow 0$ the cross section $d\left(\Delta \sigma^{(q)}\right)$ also goes to zero. We also note that the cross section (79) is not a monotonic function of $k_{T}$. In particular, for positive $k^{2}$ it starts out negative at small $k_{T}$, becoming positive for $k_{T}>Q_{s} \alpha \sqrt{1+\alpha^{2}} /\left(1-\alpha^{2}\right)$, in agreement with the large- $k_{T}$ asymptotics of Eq. (72).

\footnotetext{
${ }^{4}$ We have done the calculation without neglecting those logarithms: the resulting changes were mainly of quantitative nature, while the obtained expression was significantly more complicated than Eq. (79). Since both the expressions with and without the logarithms are approximate, we decided to only show the latter in this work due to its relative compactness.
} 


\section{The Unpolarized Cross-Section}

The real hadronic STSA in Eq. (11) contains contributions from both quark and gluon production cross sections (32) and (44) in the numerator and in the denominator, convoluted with the fragmentation functions for the quarks and gluons decaying into a particular hadron species as well as the transversity distribution of polarized quarks. This is what needs to be done to have a real comparison of the data with our theoretical results. While such comparison is beyond the scope of this work, we would like to assess the main qualitative features of our STSA-generating mechanism by concentrating on quark STSA only.

It may be tempting to consider a situation where both the numerator and the denominator of Eq. (11) are driven by the quark contributions. However, the unpolarized valence quark production cross section (32b) is known to decrease with decreasing quark momentum fraction $\alpha$ 77, 78], while both the unpolarized gluon and sea quark production cross sections grow with decreasing $\alpha$ in theoretical calculations [88, 89]. In the actual experiments the hadron multiplicity also increases as we move further away from the projectile in rapidity.

Therefore, in order to get a somewhat realistic evaluation of the qualitative behavior of the obtained STSA, we will use the unpolarized gluon production cross section in the denominator of Eq. (11). While evaluation of the unpolarized gluon cross section (44b) along the same lines as were used to obtain Eq. (79) is somewhat involved, we will approximate the result by assuming that the produced gluon is soft (i.e., far from the projectile in rapidity), in which case the corresponding production cross section is [56, 76, 87]

$$
d \sigma_{u n p}^{(G)} \approx \frac{\alpha_{s} N_{c}}{2 \pi} \int_{0}^{\infty} d x_{T}^{2}\left\{-\frac{1}{k_{T}^{2}}+\frac{2}{k_{T}^{2}} e^{-\frac{k_{T}^{2}}{Q_{s}^{2}\left(x_{T}\right)}}+\frac{1}{Q_{s}^{2}\left(x_{T}\right)} e^{-\frac{k_{T}^{2}}{Q_{s}^{2}\left(x_{T}\right)}}\left[\operatorname{Ei}\left(\frac{k_{T}^{2}}{Q_{s}^{2}\left(x_{T}\right)}\right)-\ln \frac{4 k_{T}^{2} \Lambda^{2}}{Q_{s}^{4}\left(x_{T}\right)}\right]\right\} .
$$

\section{Single Transverse Spin Asymmetry}

We now have all the essential ingredients to sketch the STSA due to quark production in the large- $N_{c}$ limit (for the wave function): we have Eqs. (79) and (80), giving the numerator and the denominator of Eq. (10) correspondingly. We thus write

$$
\begin{aligned}
A_{N}^{(q)}(\boldsymbol{k}) & =\frac{\pi^{2} N_{c} \alpha_{s}^{5} m}{4} \frac{1-\alpha}{\alpha^{4}} \int_{0}^{\infty} d x_{T}^{2}\left[T^{\prime}\left(x_{T}\right)\right]^{2} \frac{k^{2} k_{T}^{2}}{Q_{s}^{14}\left(x_{T}\right)}\left[\left(1-\alpha^{2}\right)^{2} k_{T}^{2}-\alpha^{2}\left(1+\alpha^{2}\right) Q_{s}^{2}\left(x_{T}\right)\right] e^{-\frac{k_{T}^{2}}{Q_{s}^{2}\left(x_{T}\right)}\left(1+\frac{1}{\alpha^{2}}\right)} \\
& \times\left[\int_{0}^{\infty} d y_{T}^{2}\left\{-\frac{1}{k_{T}^{2}}+\frac{2}{k_{T}^{2}} e^{-\frac{k_{T}^{2}}{Q_{s}^{2}\left(y_{T}\right)}}+\frac{1}{Q_{s}^{2}\left(y_{T}\right)} e^{-\frac{k_{T}^{2}}{Q_{s}^{2}\left(y_{T}\right)}}\left[\operatorname{Ei}\left(\frac{k_{T}^{2}}{Q_{s}^{2}\left(y_{T}\right)}\right)-\ln \frac{4 k_{T}^{2} \Lambda^{2}}{Q_{s}^{4}\left(y_{T}\right)}\right]\right\}\right]^{-1} .
\end{aligned}
$$

The $x_{T^{-}}$and $y_{T}$-integrals in Eq. (81) appear to be very hard to evaluate analytically. Instead we evaluate the integrals numerically assuming a simple Gaussian form of the nuclear profile function,

$$
T(\boldsymbol{b})=\frac{4}{3} R \rho e^{-b_{T}^{2} / R^{2}}
$$

with $R$ the nuclear radius and $\rho$ the nucleon density. Such Gaussian profiles are of course not realistic for nuclei, but have been successfully used to describe protons (see e.g. [90]).

In evaluating the STSA in Eq. (81) one has to remember that in the standard convention one has to choose $\boldsymbol{k}$ in the direction left of the beam, which, in our notation, means along the negative $y$-axis (see Fig. 1). Hence we need to replace $k^{2} \rightarrow-k_{T}$ in Eq. (81).

To plot Eq. (81) we will attempt to use somewhat realistic numbers, while realizing that all the theoreticallycalculated cross sections are likely to have non-perturbative normalization corrections, which may affect the size of the effect. To that end, we will use the saturation scale (cf. Eq. (53))

$$
Q_{s}^{2}(\boldsymbol{b})=2 \pi \alpha_{s}^{2} K^{2} T(\boldsymbol{b})
$$

with the $K$-factor fixed at $K=10$ to make $Q_{s} \approx 1 \mathrm{GeV}$, which is a realistic value for a proton. (Each $T^{\prime}\left(x_{T}\right)$ in Eq. (81) is multiplied by the same $K^{2}$-factor, since it also arises from the saturation scale.) We put $m=300 \mathrm{MeV}$ to mimic a constituent quark, along with $\rho=0.35 \mathrm{fm}^{-3}$ for a proton of radius $R=0.878 \mathrm{fm}$, and $\alpha_{s}=0.3$. We plot the resulting $A_{N}^{(q)}$ from Eq. (81) in Fig. 13 for different values of $\alpha$ with the IR cutoff $\Lambda=100 \mathrm{MeV}$ and cutting off the $x_{T^{-}}$and $y_{T}$ integrals in Eq. (81) at $2.1 \mathrm{fm}$ in the IR. (Note that strictly-speaking the CGC formalism employed 
here is valid only for scattering on a nuclear target, since it resums powers of a large parameter $\alpha_{s}^{2} A^{1 / 3}$. However its applications to proton target have been successful phenomenologically in the past [91], giving one hope that our estimates here could be relevant for $p^{\uparrow}+p$ collisions.)

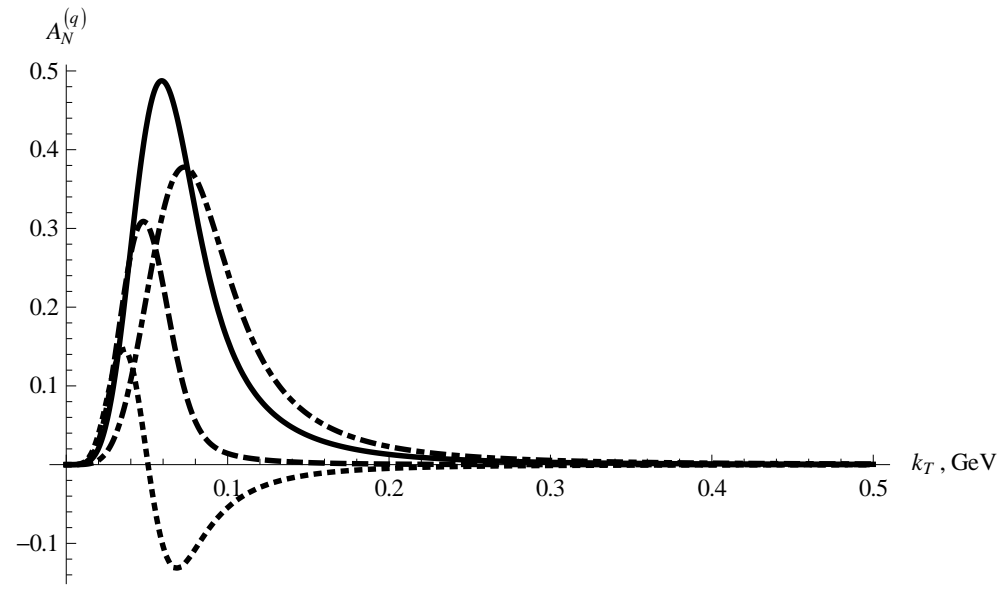

FIG. 13. Quark STSA from Eq. (81) for the proton target plotted as a function of $k_{T}$ for different values of the longitudinal momentum fraction $\alpha$ carried by the produced quark: $\alpha=0.9$ (dash-dotted curve), $\alpha=0.7$ (solid curve), $\alpha=0.6$ (dashed curve), and $\alpha=0.5$ (dotted curve).

From Fig. 13 we see that our STSA is a non-monotonic function of transverse momentum $k_{T}$, first rising and then falling off with $k_{T}$ in qualitative agreement with the data shown in the right panel of Fig. 2. As one can clearly see from Eq. (81) the maximum of $A_{N}^{(q)}$ at impact parameter $x_{T}$ in our formalism is determined (up to a constant) by the saturation scale, $k_{T} \sim Q_{s}\left(x_{T}\right)$, such that the asymmetry integrated over all impact parameters peaks at $k_{T} \sim Q_{s}$ with $Q_{s}$ an effective averaged saturation scale. The conclusion about $A_{N}$ peaking at $k_{T} \approx Q_{s}$ was previously reached in 62]. Let us stress again that the STSA in our case changes sign when plotted as a function of $k_{T}$ or $\alpha$ (i.e., it has a "node").

Note that, while the magnitude of STSA plotted in Fig. 13 can be as large as tens of percent, like the data in Fig. 2. the momentum at which the asymmetry is non-zero appears to be much smaller in our Fig. 13 than it is in the data of Fig. 2. The discrepancy of the $k_{T}$-range of the data and our Fig. 13 signals the following potential problem: the $x_{T}$-integral in Eq. (81) is dominated by large $x_{T}$, where $Q_{s}\left(x_{T}\right)$ is small, leading to small values of $k_{T}$ dominating $A_{N}$, and potentially making the corresponding physics non-perturbative. Thus our perturbative calculation appears to be sensitive to the non-perturbative domain.

To illustrate the range of spectra that can be obtained by our estimates, we replot $A_{N}$ from Fig. 13 in Fig. 14 cutting off the $x_{T^{-}}$and $y_{T}$-integrals in Eq. (81) by $1.3 \mathrm{fm}$. In addition, we mimic the coordinate-space logarithms, like those that were neglected after (75), by introducing a factor of $\ln k_{T} / \tilde{m}$. In this plot the $k_{T}$-range of the asymmetry is broader than in Fig. 13, which makes it closer to the experimental data in Fig. 2, but the height of the asymmetry is over an order-of-magnitude lower than the data. More work is needed to assess whether the cutoff dependence is a result of the approximations made, or whether it actually signals a potential breakdown of the approach indicating the non-perturbative nature of STSA.

Another important observation one can make from Fig. 13 is that $A_{N}^{(q)}$ increases with increasing $\alpha$, except for very large values of $\alpha$ when it starts to decrease. The increase of $A_{N}^{(q)}$ with increasing $\alpha$ is in qualitative agreement with the data in the left panel of Fig. 2, where the data points increase with increasing Feynman- $x$. One can also see that while our STSA in Fig. 13 is mostly positive, the data for $\pi^{-}$mesons in the left panel of Fig. 2 gives a negative STSA. This result can be explained in the following simple model. Imagine a constituent-quark model of a proton, with the spins of both up quarks aligned with the net proton spin, and the spin of the down quark pointing in the opposite direction. Then in our mechanism each up-quark would give a positive STSA denoted $A_{u}$, while the down-quark would give a negative STSA of the same absolute value, denoted $A_{d}=-A_{u}$. Imagining that after the collision the up-quarks fragment into $\pi^{+}$'s and $\pi^{0}$ 's, while the down quarks fragment into $\pi^{0}$ 's and $\pi^{-}$'s, and taking into account that there are twice as many up-quarks than down-quarks in the proton, and neglecting pions resulting from the gluon fragmentation, we obtain $A_{\pi^{+}}=A_{u}, A_{\pi^{0}}=A_{u} / 2$, and $A_{\pi^{-}}=A_{d} / 2=-A_{u} / 2$, thus obtaining a negative STSA for 


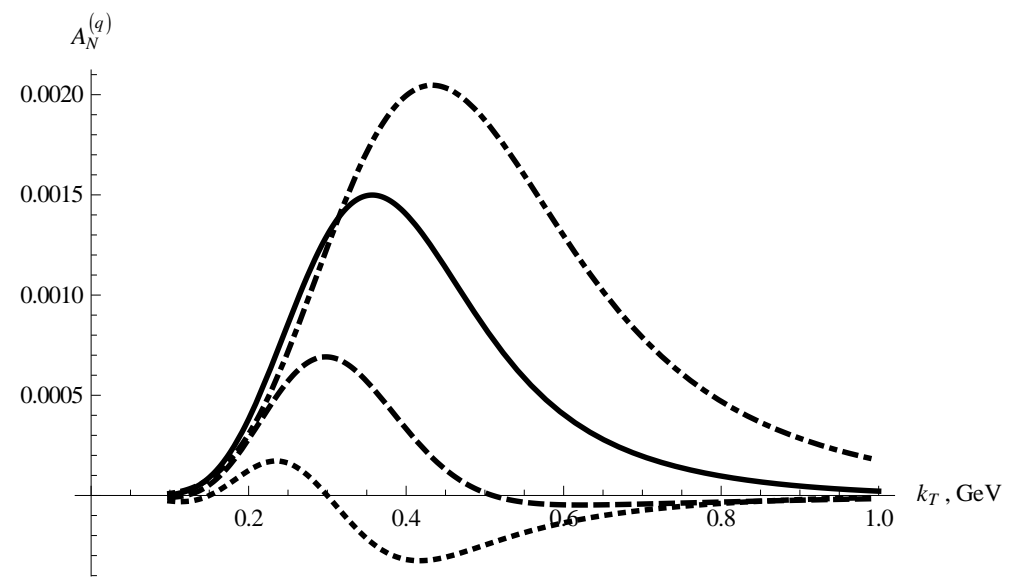

FIG. 14. Same as in Fig. 13 but with the $1.3 \mathrm{fm}$ upper cutoff on the $x_{T^{-}}$and $y_{T}$ integrals in Eq. (81) and a factor of $\ln k_{T} / \tilde{m}$ inserted.

$\pi^{-}$s: 5 This naive model appears to be in a qualitative agreement (and in loose quantitative agreement) with the data in the left panel of Fig. 2. Inclusion of gluon fragmentation may further improve the quantitative agreement with the data.

Finally, to test the dependence of our STSA in Eq. (81) on the size of the target, we note that for $k_{T} \approx Q_{s}$ one gets

$$
A_{N}^{(q)}\left(k_{T} \approx Q_{s}\right) \sim \frac{1}{Q_{s}^{7}} \sim A^{-7 / 6},
$$

if $Q_{s}^{2} \sim A^{1 / 3}$. This indicates a very steep falloff of STSA with the atomic number of the nuclear target. Such a conclusion appears to be supported by the numerical evaluation of Eq. (81) for several different radii of the target shown in Fig. 15. (Now the $x_{T^{-}}$and $y_{T}$ integrals are cut off at $2.4 \mathrm{fm}$.) One can see that $A_{N}^{(q)}$ drops very rapidly with the size of the target. If the experimentally observed STSA in $p^{\uparrow}+p$ collisions are due to our mechanism, our

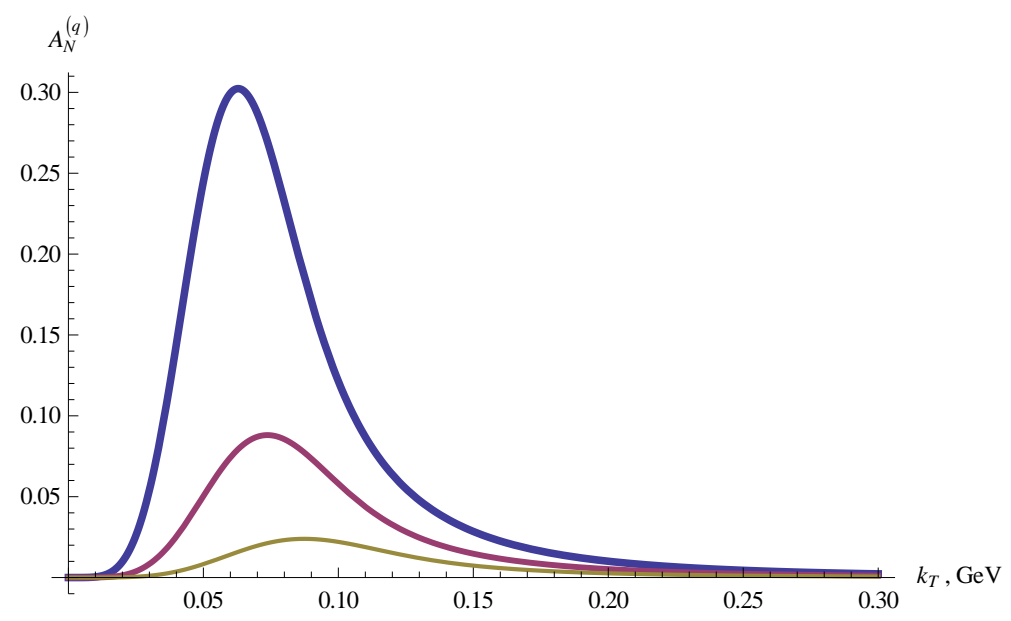

FIG. 15. Quark STSA from Eq. (81) plotted as a function of $k_{T}$ for different values of the target radius: $R=1 \mathrm{fm}$ (top curve), $R=1.4 \mathrm{fm}$ (middle curve), and $R=2 \mathrm{fm}$ (bottom curve) for $\alpha=0.7$.

prediction is then that in $p^{\uparrow}+A$ collisions STSA should be much smaller than that in $p^{\uparrow}+p$. In the case of a heavy ion target like $A u$ the STSA due to our mechanism is likely to be negligibly small.

While we have demonstrated here the potential for our calculations to agree with the data, the evaluations presented here have to be significantly improved to reach a definitive conclusion. For instance the $k_{T}$-dependence and the overall

\footnotetext{
${ }^{5}$ We would like to thank Mickey Chiu for a discussion of these estimates.
} 
normalization in our Eqs. (79) and (80) are overly simple and is not ready to be compared to the data. The equations need to be corrected for the effects of DGLAP evolution, small- $x$ evolution and for the running of the coupling for a meaningful quantitative comparison with the data. Only such a phenomenological analysis can determine whether our mechanism for generating STSA is dominant, or whether it is simply one of the many factors contributing to the asymmetry.

\section{B. STSA in Photon Production}

Using the methods developed in Sec. II A we can now evaluate the photon STSA given by Eqs. (49) and (51). Substituting Eq. (51b) into Eq. (49a) and performing the variable shift of Eq. (61) while keeping in mind that now $\boldsymbol{u}$ and $\boldsymbol{w}$ are given by Eqs. (46) yields

$$
d\left(\Delta \sigma^{(\gamma)}\right)=\frac{i}{(2 \pi)^{3}} \int d^{2} x d^{2} y d^{2} z e^{-i \boldsymbol{k} \cdot(\boldsymbol{z}-\boldsymbol{y})} \Phi_{p o l}(-\boldsymbol{z},-\boldsymbol{y}, \alpha)\left[O_{\boldsymbol{x}+(1-\alpha) \boldsymbol{z}, \boldsymbol{x}+(1-\alpha) \boldsymbol{y}}-O_{\boldsymbol{x}, \boldsymbol{x}+(1-\alpha) \boldsymbol{y}}-O_{\boldsymbol{x}+(1-\alpha) \boldsymbol{z}, \boldsymbol{x}}\right]
$$

where we have again dropped the tildes for brevity. Using the argument of Eqs. (65) and (66) we see that each term in the square brackets in Eq. (85) is zero after the integration over $\boldsymbol{x}$. We thus have an exact result that

$$
d\left(\Delta \sigma^{(\gamma)}\right)=0
$$

in our mechanism for generating photon STSA. Hence the photon STSA is zero, $A_{N}^{(\gamma)}=0$, in the forward production region under consideration.

\section{CONCLUSIONS}

To conclude let us summarize the main points of this work. Above we have shown how STSA can be generated in the CGC formalism for quark and gluon production. The results for the corresponding cross sections are given in Eqs. (32), (40) and (44). The same mechanism gives zero STSA for prompt photons.

In our case STSA is generated by both a splitting in the projectile wave function, and by the combination of the $C$-odd and $C$-even interactions with the target. Hence our STSA-generating mechanism is distinctively different from the Collins [27] and Sivers [15, 16] effects, and is more akin to (though still different from) the higher-twist mechanisms of $17-26]$.

Evaluating the quark STSA in a simplified quasi-classical model we found qualitative agreement with the data: quark STSA appears to be a non-monotonic function of $k_{T}$, and is an increasing function of increasing $x_{F}$ (for most of the $x_{F}$-range). It is perhaps encouraging that the obtained asymmetry can be of the order-of-magnitude of the experimental data. Further phenomenological studies of our formulas (32) and (44) are needed to determine whether this qualitative agreement of our results with the data can become quantitative.

Analyzing the general quark production formula (27) one can see that the contribution to STSA arises from the $\boldsymbol{z} \leftrightarrow \boldsymbol{y}$ anti-symmetric part of the integrand. In arriving at Eq. (32a) from Eq. (27) we employed the lowest-order (order- $\alpha_{s}$ ) spin-dependent part of the light-cone wave function squared (15), which happens to be symmetric under the $\boldsymbol{z} \leftrightarrow \boldsymbol{y}$ interchange: hence, in our case, to obtain a contribution to the STSA the interaction with the target had to be $\boldsymbol{z} \leftrightarrow \boldsymbol{y}$ anti-symmetric. However, it is possible that higher-order corrections to the light-cone wave function squared would lead to a $\boldsymbol{z} \leftrightarrow \boldsymbol{y}$ anti-symmetric contribution. (By the 'wave function corrections' we understand all the initial and final state corrections with rapidities between the projectile and the particle we tag on.) In such case, the interaction with the target need not be $\boldsymbol{z} \leftrightarrow \boldsymbol{y}$ anti-symmetric, and can be mediated by the standard $C$-even exchange. To test whether such scenario is feasible within the CGC/saturation perturbative framework one has to calculate the higher order corrections to the polarization-dependent light-cone wave function squared (15). The corrections would need a generate a relative complex phase between the corrected and uncorrected wave functions, in agreement with the initial proposal of [22, 23]. This can be accomplished in LCPT if the corrections lead to an intermediate state, for which the imaginary part of the energy denominator leads to a non-vanishing polarization-dependent contribution to the scattering amplitude. An example of such corrections in our case could be a modification of the amplitude in Fig. 6 resulting from a gluon exchange between the outgoing quark and gluon formed in the projectile splitting. Calculation of such diagrams appears to be rather complicated and is beyond the scope of this work. However, it potentially may give a contribution comparable to the STSA resulting from Eq. (32a): the latter consists of the order- $\alpha_{s}$ light-cone wave function squared, convoluted with the target interaction resumming powers of $\alpha_{s}^{2} A^{1 / 3}$ and $\alpha_{s} Y$, with one extra power of $\alpha_{s}$ due to the odderon exchange (56). Our contribution (32a) is, therefore, order- $\alpha_{s}^{2}$, 
if one assumes that $\alpha_{s}^{2} A^{1 / 3} \sim 1$ and $\alpha_{s} Y \sim 1$, which is parametrically comparable to the $C$-odd order- $\alpha_{s}^{2}$ light-cone wave function squared, interacting with the target through a $C$-even order-one exchange. An explicit calculation is needed to explore this possibility and is left for future work.

\section{ACKNOWLEDGMENTS}

The authors are much indebted to Mike Lisa for strongly encouraging them to think about spin physics at RHIC. We would like to also thank Mickey Chiu, Alex Kovner, and Genya Levin for stimulating discussions on the subject, and Daniel Boer, John Collins, Jianwei Qiu, and Raju Venugopalan for comments on the manuscript. M.D.S. would like to thank the Department of Energy's Institute for Nuclear Theory at the University of Washington for the hospitality and constructive atmosphere that facilitated this work.

This research is sponsored in part by the U.S. Department of Energy under Grant No. de-sc0004286

\section{Appendix A: Averaging the odderon amplitude over target wave function}

Let us construct the dipole odderon amplitude averaged over the target field. The triple gluon exchange happens between the dipole and a nucleon in the target, which, for simplicity we model as a valence quark in a bag. (The overall factor in front of the averaged odderon amplitude should indeed depend on the details of the averaging; however, we believe the coordinate-space dependence would remain the same for other models of the nuclear wave function.) The target averaging then consists of averaging over the positions of the quark in the nucleon and over the positions of nucleons in the nucleus, along with summation over all nucleons. Assuming, again for simplicity, that the quark has equal probability to be anywhere inside the nucleon in the transverse plane (a cylindrical "nucleon" approximation), we write for the averaged odderon amplitude

$$
\begin{aligned}
& O_{\boldsymbol{x} \boldsymbol{y}}=c_{0} \alpha_{s}^{3} \int d^{2} b T(\boldsymbol{b}) \int \frac{d^{2} r}{\pi a^{2}} \ln ^{3} \frac{|\boldsymbol{x}-\boldsymbol{b}-\boldsymbol{r}|}{|\boldsymbol{y}-\boldsymbol{b}-\boldsymbol{r}|} \theta(a-r) \theta\left(a-\left|\frac{\boldsymbol{x}+\boldsymbol{y}}{2}-\boldsymbol{b}\right|\right) \\
& \times \exp \left[-\frac{1}{4}|\boldsymbol{x}-\boldsymbol{y}|^{2} Q_{s}^{2}\left(\frac{\boldsymbol{x}+\boldsymbol{y}}{2}\right) \ln \frac{1}{|\boldsymbol{x}-\boldsymbol{y}| \Lambda}\right] .
\end{aligned}
$$

Here $\boldsymbol{b}$ is the position of the center of a nucleon in the transverse plane with respect to the center of the nucleus, $\boldsymbol{r}$ is the position of the valence quark in the nucleon, and $a$ is the radius of the nucleon, as illustrated in Fig. 16. The two theta-functions in Eq. (A1) insure that the valence quark and the center of the $\boldsymbol{x}, \boldsymbol{y}$-dipole are both located inside the nucleon in the transverse plane. In our simple model of the collision the dipole has to hit the nucleon directly in

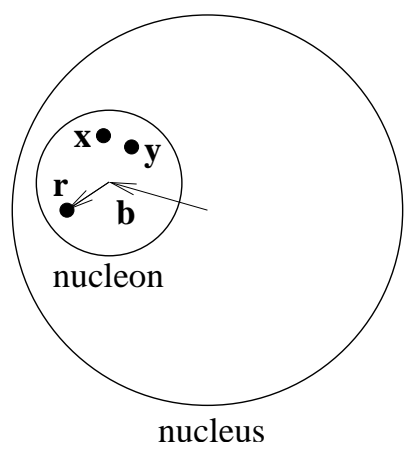

FIG. 16. The geometry of the dipole-nucleus scattering as employed in Eq. A1.

order to be able to interact with the quarks inside of it. Since the dipole $\boldsymbol{x}, \boldsymbol{y}$ is perturbatively small, we enforce this condition by demanding that only the center of the dipole is inside the nucleon's transverse extent.

To integrate over $\boldsymbol{r}$ in Eq. A1 we notice that

$$
\int d^{2} r \ln ^{3} \frac{|\boldsymbol{x}-\boldsymbol{b}-\boldsymbol{r}|}{|\boldsymbol{y}-\boldsymbol{b}-\boldsymbol{r}|}=0
$$

if the integration carries over the whole transverse plane. Using this result we write

$$
\int d^{2} r \ln ^{3}\left(\frac{|\boldsymbol{x}-\boldsymbol{b}-\boldsymbol{r}|}{|\boldsymbol{y}-\boldsymbol{b}-\boldsymbol{r}|}\right) \theta(a-r)=-\int d^{2} r \ln ^{3}\left(\frac{|\boldsymbol{x}-\boldsymbol{b}-\boldsymbol{r}|}{|\boldsymbol{y}-\boldsymbol{b}-\boldsymbol{r}|}\right) \theta(r-a) .
$$


To approximate the integral on the right-hand-side of Eq. (A3) we expand its integrand in powers of $|\boldsymbol{x}-\boldsymbol{b}| / r$ and $|\boldsymbol{y}-\boldsymbol{b}| / r$ to the first non-trivial (after integration) order, thus obtaining

$$
\int d^{2} r \ln ^{3}\left(\frac{|\boldsymbol{x}-\boldsymbol{b}-\boldsymbol{r}|}{|\boldsymbol{y}-\boldsymbol{b}-\boldsymbol{r}|}\right) \theta(a-r) \approx \frac{3 \pi}{8 a^{2}}|\boldsymbol{x}-\boldsymbol{y}|^{2}(\boldsymbol{x}-\boldsymbol{y}) \cdot(\boldsymbol{x}+\boldsymbol{y}-2 \boldsymbol{b}) .
$$

Substituting Eq. (A4) back into Eq. (A1) and defining a new integration variable

$$
\tilde{b}=\boldsymbol{b}-\frac{\boldsymbol{x}+\boldsymbol{y}}{2}
$$

yields

$$
\begin{array}{r}
O_{\boldsymbol{x} \boldsymbol{y}} \approx-c_{0} \alpha_{s}^{3} \frac{3}{4 a^{4}}|\boldsymbol{x}-\boldsymbol{y}|^{2} \exp \left[-\frac{1}{4}|\boldsymbol{x}-\boldsymbol{y}|^{2} Q_{s}^{2}\left(\frac{\boldsymbol{x}+\boldsymbol{y}}{2}\right) \ln \frac{1}{|\boldsymbol{x}-\boldsymbol{y}| \Lambda}\right] \\
\times(\boldsymbol{x}-\boldsymbol{y}) \cdot \int d^{2} \tilde{b} \tilde{\boldsymbol{b}} T\left(\frac{\boldsymbol{x}+\boldsymbol{y}}{2}+\tilde{\boldsymbol{b}}\right) \theta\left(a-\tilde{b}_{T}\right) .
\end{array}
$$

In principle this result is as far as one can simplify $O_{x y}$ without the explicit knowledge of the nuclear profile function $T(\boldsymbol{b})$. To obtain a closed expression for the STSA in the text we expand

$$
T\left(\frac{\boldsymbol{x}+\boldsymbol{y}}{2}+\tilde{\boldsymbol{b}}\right)=T\left(\frac{\boldsymbol{x}+\boldsymbol{y}}{2}\right)+\tilde{\boldsymbol{b}} \cdot \boldsymbol{\nabla} T\left(\frac{\boldsymbol{x}+\boldsymbol{y}}{2}\right)+\ldots
$$

with $\boldsymbol{\nabla}$ the transverse gradient operator. Such expansion is potentially dangerous near the edge of the nucleus profile, where the derivatives may get large. For instance, for a solid-sphere model of the nucleus the nuclear profile function is $T(\boldsymbol{b})=\rho 2 \sqrt{R^{2}-b^{2}}$ with $\rho$ the nucleon density and $R$ the nuclear radius; the derivatives of such $T(\boldsymbol{b})$ near $b=R$ are divergent. Using the realistic Woods-Saxon profile would make the derivatives finite, but they would still be large. Thus we will proceed by using the expansion (A7) as a way to simplify the expression, keeping in mind that in the cases where this expansion breaks down one has to return back to Eq. A6.

Substituting Eq. (A7) into Eq. (A6) and integrating over $\tilde{\boldsymbol{b}}$ yields (for the first non-trivial term after integration)

$$
O_{\boldsymbol{x} y} \approx-c_{0} \alpha_{s}^{3} \frac{3 \pi}{16}|\boldsymbol{x}-\boldsymbol{y}|^{2} \exp \left[-\frac{1}{4}|\boldsymbol{x}-\boldsymbol{y}|^{2} Q_{s}^{2}\left(\frac{\boldsymbol{x}+\boldsymbol{y}}{2}\right) \ln \frac{1}{|\boldsymbol{x}-\boldsymbol{y}| \Lambda}\right](\boldsymbol{x}-\boldsymbol{y}) \cdot \nabla T\left(\frac{\boldsymbol{x}+\boldsymbol{y}}{2}\right) .
$$

This is our final expression for the target-averaged odderon amplitude. Note an interesting feature of Eq. (A8): the non-zero contribution to the odderon amplitude in the transverse coordinate space arises from the gradient of the nuclear profile function. The odderon interaction with the target is thus only possible if the target has a non-uniform profile in the transverse space. This is in stark contrast to the $C$-even exchanges, which are non-zero even for the Bjorken model of a nucleus of infinite transverse extent with constant density in the transverse plane.

[1] U. D'Alesio and F. Murgia, Azimuthal and Single Spin Asymmetries in Hard Scattering Processes, Prog.Part.Nucl.Phys. 61 (2008) 394-454, arXiv:0712.4328. Invited review paper to be published in Prog.Part.Nucl.Phys.

[2] E581, E704 Collaboration, D. Adams et. al., Comparison of spin asymmetries and cross-sections in pi0 production by 200-GeV polarized anti-protons and protons, Phys.Lett. B261 (1991) 201-206.

[3] FNAL-E704 Collaboration, D. Adams et. al., Analyzing power in inclusive pi+ and pi-production at high $x(F)$ with a 200-GeV polarized proton beam, Phys.Lett. B264 (1991) 462-466.

[4] E581, E704 Collaboration, D. Adams et. al., Large $x(F)$ spin asymmetry in pi0 production by 200-GeV polarized protons, Z.Phys. C56 (1992) 181-184.

[5] E704 Collaboration, D. Adams et. al., Measurement of single spin asymmetry for direct photon production in $p$ p collisions at 200-GeV/c, Phys.Lett. B345 (1995) 569-575.

[6] Fermilab E704 Collaboration, A. Bravar et. al., Single spin asymmetries in inclusive charged pion production by transversely polarized anti-protons, Phys.Rev.Lett. 77 (1996) 2626-2629.

[7] FNAL E704 Collaboration, D. Adams et. al., Single spin asymmetries and invariant cross-sections of the high transverse momentum inclusive piO production in 200-GeV/c p p and anti-p p interactions, Phys.Rev. D53 (1996) 4747-4755.

[8] STAR Collaboration, B. Abelev et. al., Measurement of transverse single-spin asymmetries for di-jet production in proton-proton collisions at $s^{* *}(1 / 2)=200-G e V$, Phys.Rev.Lett. 99 (2007) 142003, arXiv:0705.4629.

[9] STAR Collaboration, L. Nogach, Measurements of Transverse Spin Effects with the Forward Pion Detector of STAR, AIP Conf.Proc. 915 (2007) 543-546, hep-ex/0612030. 
[10] PHENIX Collaboration, S. Adler et. al., Measurement of transverse single-spin asymmetries for mid-rapidity production of neutral pions and charged hadrons in polarized $p+p$ collisions at $s^{* *}(1 / 2)=200-G e V$, Phys.Rev.Lett. 95 (2005) 202001, hep-ex/0507073.

[11] BRAHMS Collaboration, J. Lee and F. Videbaek, Single spin asymmetries of identified hadrons in polarized $p+p$ at $s^{* *}(1 / 2)=62.4$ and 200-GeV, AIP Conf.Proc. 915 (2007) 533-538.

[12] STAR Collaboration, B. Abelev et. al., Forward Neutral Pion Transverse Single Spin Asymmetries in $p+p$ Collisions at $s^{* *}(1 / 2)=200-G e V$, Phys.Rev.Lett. 101 (2008) 222001, arXiv:0801.2990.

[13] PHENIX Collaboration, F. Wei, Transverse Spin Results From PHENIX, arXiv:1108.0911.

[14] G. L. Kane, J. Pumplin, and W. Repko, Transverse Quark Polarization in Large p(T) Reactions, e+ e- Jets, and Leptoproduction: A Test of QCD, Phys.Rev.Lett. 41 (1978) 1689.

[15] D. W. Sivers, Single Spin Production Asymmetries from the Hard Scattering of Point-Like Constituents, Phys.Rev. D41 (1990) 83.

[16] D. W. Sivers, Hard scattering scaling laws for single spin production asymmetries, Phys.Rev. D43 (1991) $261-263$.

[17] A. Efremov and O. Teryaev, ON SPIN EFFECTS IN QUANTUM CHROMODYNAMICS, Sov.J.Nucl.Phys. 36 (1982) 140.

[18] A. Efremov and O. Teryaev, QCD Asymmetry and Polarized Hadron Structure Functions, Phys.Lett. B150 (1985) 383.

[19] J.-w. Qiu and G. F. Sterman, Single transverse spin asymmetries, Phys.Rev.Lett. 67 (1991) $2264-2267$.

[20] X.-D. Ji, Gluon correlations in the transversely polarized nucleon, Phys.Lett. B289 (1992) 137-142.

[21] J.-w. Qiu and G. F. Sterman, Single transverse spin asymmetries in hadronic pion production, Phys.Rev. D59 (1998) 014004, hep-ph/9806356.

[22] S. J. Brodsky, D. S. Hwang, and I. Schmidt, Final state interactions and single spin asymmetries in semiinclusive deep inelastic scattering, Phys.Lett. B530 (2002) 99-107, hep-ph/0201296.

[23] J. C. Collins, Leading twist single transverse-spin asymmetries: Drell-Yan and deep inelastic scattering, Phys.Lett. B536 (2002) 43-48, hep-ph/0204004.

[24] Y. Koike and S. Yoshida, Probing the three-gluon correlation functions by the single spin asymmetry in $p^{\uparrow} p \rightarrow D X$, Phys.Rev. D84 (2011) 014026, arXiv:1104.3943.

[25] Y. Kanazawa and Y. Koike, Chiral odd contribution to single transverse spin asymmetry in hadronic pion production, Phys.Lett. B478 (2000) 121-126, hep-ph/0001021.

[26] Y. Kanazawa and Y. Koike, Estimate of a chiral odd contribution to single transverse spin asymmetry in hadronic pion production, Phys.Lett. B490 (2000) 99-105, hep-ph/0007272.

[27] J. C. Collins, Fragmentation of transversely polarized quarks probed in transverse momentum distributions, Nucl.Phys. B396 (1993) 161-182, hep-ph/9208213.

[28] STAR Collaboration, N. Poljak, Forward jet-like event spin-dependent properties in polarized $p+p$ collisions at $\sqrt{s}=200$ GeV, J.Phys.Conf.Ser. 295 (2011) 012102, arXiv:1012.0221.

[29] L. V. Gribov, E. M. Levin, and M. G. Ryskin, Semihard Processes in QCD, Phys. Rept. 100 (1983) 1-150.

[30] J. P. Blaizot and A. H. Mueller, The Early Stage of Ultrarelativistic Heavy Ion Collisions, Nucl. Phys. B289 (1987) 847.

[31] A. H. Mueller and J.-w. Qiu, Gluon recombination and shadowing at small values of x, Nucl. Phys. B268 (1986) 427.

[32] A. H. Mueller, Soft gluons in the infinite momentum wave function and the BFKL pomeron, Nucl. Phys. B415 (1994) 373-385.

[33] A. H. Mueller and B. Patel, Single and double BFKL pomeron exchange and a dipole picture of high-energy hard processes, Nucl. Phys. B425 (1994) 471-488, hep-ph/9403256.

[34] A. H. Mueller, Unitarity and the BFKL pomeron, Nucl. Phys. B437 (1995) 107-126, hep-ph/9408245].

[35] L. D. McLerran and R. Venugopalan, Gluon distribution functions for very large nuclei at small transverse momentum, Phys. Rev. D49 (1994) 3352-3355, hep-ph/9311205.

[36] L. D. McLerran and R. Venugopalan, Computing quark and gluon distribution functions for very large nuclei, Phys. Rev. D49 (1994) 2233-2241, hep-ph/9309289.

[37] L. D. McLerran and R. Venugopalan, Green's functions in the color field of a large nucleus, Phys. Rev. D50 (1994) 2225-2233, hep-ph/9402335.

[38] Y. V. Kovchegov, Non-abelian Weizsaecker-Williams field and a two-dimensional effective color charge density for a very large nucleus, Phys. Rev. D54 (1996) 5463-5469, hep-ph/9605446.

[39] Y. V. Kovchegov, Quantum structure of the non-abelian Weizsaecker-Williams field for a very large nucleus, Phys. Rev. D55 (1997) 5445-5455, hep-ph/9701229.

[40] J. Jalilian-Marian, A. Kovner, L. D. McLerran, and H. Weigert, The intrinsic glue distribution at very small $x$, Phys. Rev. D55 (1997) 5414-5428, hep-ph/9606337.

[41] J. Jalilian-Marian, A. Kovner, A. Leonidov, and H. Weigert, The BFKL equation from the Wilson renormalization group, Nucl. Phys. B504 (1997) 415-431, hep-ph/9701284.

[42] J. Jalilian-Marian, A. Kovner, A. Leonidov, and H. Weigert, The Wilson renormalization group for low $x$ physics: Towards the high density regime, Phys. Rev. D59 (1998) 014014, hep-ph/9706377.

[43] J. Jalilian-Marian, A. Kovner, and H. Weigert, The Wilson renormalization group for low x physics: Gluon evolution at finite parton density, Phys. Rev. D59 (1998) 014015, hep-ph/9709432.

[44] J. Jalilian-Marian, A. Kovner, A. Leonidov, and H. Weigert, Unitarization of gluon distribution in the doubly logarithmic regime at high density, Phys. Rev. D59 (1999) 034007, hep-ph/9807462.

[45] A. Kovner, J. G. Milhano, and H. Weigert, Relating different approaches to nonlinear QCD evolution at finite gluon density, Phys. Rev. D62 (2000) 114005, hep-ph/0004014. 
[46] H. Weigert, Unitarity at small Bjorken x, Nucl. Phys. A703 (2002) 823-860, hep-ph/0004044.

[47] E. Iancu, A. Leonidov, and L. D. McLerran, Nonlinear gluon evolution in the color glass condensate. I, Nucl. Phys. A692 (2001) 583-645, hep-ph/0011241.

[48] E. Ferreiro, E. Iancu, A. Leonidov, and L. McLerran, Nonlinear gluon evolution in the color glass condensate. II, Nucl. Phys. A703 (2002) 489-538, hep-ph/0109115.

[49] I. Balitsky, Operator expansion for high-energy scattering, Nucl. Phys. B463 (1996) 99-160, hep-ph/9509348.

[50] I. Balitsky, Operator expansion for diffractive high-energy scattering, hep-ph/9706411.

[51] I. Balitsky, Factorization and high-energy effective action, Phys. Rev. D60 (1999) 014020, hep-ph/9812311.

[52] Y. V. Kovchegov, Small-x $F_{2}$ structure function of a nucleus including multiple pomeron exchanges, Phys. Rev. D60 (1999) 034008, hep-ph/9901281.

[53] Y. V. Kovchegov, Unitarization of the BFKL pomeron on a nucleus, Phys. Rev. D61 (2000) 074018, hep-ph/9905214.

[54] E. Iancu and R. Venugopalan, The color glass condensate and high energy scattering in QCD, hep-ph/0303204.

[55] H. Weigert, Evolution at small $x_{b j}$ : The Color Glass Condensate, Prog. Part. Nucl. Phys. 55 (2005) 461-565, hep-ph/0501087.

[56] J. Jalilian-Marian and Y. V. Kovchegov, Saturation physics and deuteron gold collisions at RHIC, Prog. Part. Nucl. Phys. 56 (2006) 104-231, hep-ph/0505052.

[57] G. P. Lepage and S. J. Brodsky, Exclusive processes in perturbative quantum chromodynamics, Phys. Rev. D22 (1980) 2157.

[58] S. J. Brodsky, H.-C. Pauli, and S. S. Pinsky, Quantum chromodynamics and other field theories on the light cone, Phys. Rept. 301 (1998) 299-486, hep-ph/9705477.

[59] J. Bartels, B. Ermolaev, and M. Ryskin, Nonsinglet contributions to the structure function g1 at small x, Z.Phys. C70 (1996) 273-280, hep-ph/9507271.

[60] A. Metz and J. Zhou, Distribution of linearly polarized gluons inside a large nucleus, Phys.Rev. D84 (2011) 051503, arXiv:1105.1991.

[61] F. Dominguez, J.-W. Qiu, B.-W. Xiao, and F. Yuan, On the linearly polarized gluon distributions in the color dipole model, Phys.Rev. D85 (2012) 045003, arXiv:1109.6293.

[62] D. Boer, A. Dumitru, and A. Hayashigaki, Single transverse-spin asymmetries in forward pion production at high energy: Incorporating small-x effects in the target, Phys.Rev. D74 (2006) 074018, hep-ph/0609083.

[63] D. Boer and A. Dumitru, Polarized hyperons from pA scattering in the gluon saturation regime, Phys.Lett. B556 (2003) $33-40$, hep-ph/0212260.

[64] D. Boer, A. Utermann, and E. Wessels, The Saturation scale and its $x$-dependence from Lambda polarization studies, Phys.Lett. B671 (2009) 91-98, arXiv:0811.0998.

[65] Z.-B. Kang and F. Yuan, Single Spin Asymmetry Scaling in the Forward Rapidity Region at RHIC, Phys. Rev. D84 (2011) 034019, arXiv:1106.1375.

[66] Y. Qian and I. Zahed, Single Spin Asymmetry through QCD Instantons, arXiv:1112.4552

[67] L. Lukaszuk and B. Nicolescu, A Possible interpretation of p p rising total cross-sections, Lett.Nuovo Cim. 8 (1973) 405-413.

[68] B. Nicolescu, The Odderon today, Presented at the Moriond 1990 Conference (1990).

[69] C. Ewerz, The Odderon in quantum chromodynamics, hep-ph/0306137.

[70] J. Bartels, L. Lipatov, and G. Vacca, A New odderon solution in perturbative QCD, Phys.Lett. B477 (2000) 178-186, hep-ph/9912423.

[71] Y. V. Kovchegov, L. Szymanowski, and S. Wallon, Perturbative odderon in the dipole model, Phys.Lett. B586 (2004) 267-281, hep-ph/0309281. Dedicated to the memory of Jan Kwiecinski.

[72] Y. Hatta, E. Iancu, K. Itakura, and L. McLerran, Odderon in the color glass condensate, Nucl.Phys. A760 (2005) 172-207, hep-ph/0501171.

[73] A. Kovner and M. Lublinsky, Odderon and seven Pomerons: QCD Reggeon field theory from JIMWLK evolution, JHEP 0702 (2007) 058, hep-ph/0512316.

[74] S. Jeon and R. Venugopalan, A Classical Odderon in QCD at high energies, Phys.Rev. D71 (2005) 125003, hep-ph/0503219.

[75] A. H. Mueller, Small x Behavior and Parton Saturation: A QCD Model, Nucl. Phys. B335 (1990) 115.

[76] Y. V. Kovchegov and A. H. Mueller, Gluon production in current nucleus and nucleon nucleus collisions in a quasi-classical approximation, Nucl. Phys. B529 (1998) 451-479, hep-ph/9802440.

[77] K. Itakura, Y. V. Kovchegov, L. McLerran, and D. Teaney, Baryon stopping and valence quark distribution at small x, Nucl. Phys. A730 (2004) 160-190, hep-ph/0305332.

[78] J. L. Albacete and Y. V. Kovchegov, Baryon stopping in proton nucleus collisions, Nucl. Phys. A781 (2007) 122-149, hep-ph/0605053.

[79] J. Cortes, B. Pire, and J. Ralston, Measuring the transverse polarization of quarks in the proton, Z.Phys. C55 (1992) 409-416.

[80] J. Bartels, High-Energy Behavior in a Nonabelian Gauge Theory. 1. T $(n \rightarrow m)$ in the Leading Log Normal $S$ Approximation, Nucl.Phys. B151 (1979) 293.

[81] J. Kwiecinski and M. Praszalowicz, Three Gluon Integral Equation and Odd c Singlet Regge Singularities in QCD, Phys.Lett. B94 (1980) 413.

[82] A. Ahmedov, I. V. Akushevich, E. A. Kuraev, and P. G. Ratcliffe, Single spin asymmetries for small angle pion production in high-energy hadron collisions, Eur.Phys.J. C11 (1999) 703-708, hep-ph/9902418. 
[83] M. Jarvinen, Single-spin asymmetry from pomeron-odderon interference, Czech.J.Phys. 56 (2006) F209-F214, hep-ph/0610101.

[84] E. Leader and T. Trueman, The Odderon and spin dependence of high-energy proton proton scattering, Phys.Rev. D61 (2000) 077504, hep-ph/9908221.

[85] N. H. Buttimore, B. Kopeliovich, E. Leader, J. Soffer, and T. Trueman, The spin dependence of high-energy proton scattering, Phys.Rev. D59 (1999) 114010, hep-ph/9901339.

[86] T. Trueman, Spin asymmetries for elastic proton scattering and the spin dependent couplings of the Pomeron, Phys.Rev. D77 (2008) 054005, arXiv:0711.4593.

[87] D. Kharzeev, Y. V. Kovchegov, and K. Tuchin, Cronin effect and high-p(t) suppression in p a collisions, Phys. Rev. D68 (2003) 094013, hep-ph/0307037.

[88] Y. V. Kovchegov and K. Tuchin, Inclusive gluon production in dis at high parton density, Phys. Rev. D65 (2002) 074026 , hep-ph/0111362.

[89] Y. V. Kovchegov and K. Tuchin, Production of q anti-q pairs in proton-nucleus collisions at high energies, Phys.Rev. D74 (2006) 054014, hep-ph/0603055.

[90] A. L. Ayala, M. B. Gay Ducati, and E. M. Levin, Qcd evolution of the gluon density in a nucleus, Nucl. Phys. B493 (1997) 305-353, hep-ph/9604383.

[91] J. L. Albacete, N. Armesto, J. G. Milhano, P. Quiroga-Arias, and C. A. Salgado, AAMQS: A non-linear QCD analysis of new HERA data at small-x including heavy quarks, Eur. Phys. J. C71 (2011) 1705, arXiv:1012.4408. 